OPEN ACCESS

Edited by:

Joseph M. Castellano, Icahn School of Medicine at Mount Sinai, United States

Reviewed by:

Jason Ulrich,

Washington University in St. Louis,

United States

Jessica Elaine Young, University of Washington,

United States

*Correspondence:

Rik van der Kant r.h.n.vander.kant@vu.n

Received: 02 April 2021 Accepted: 28 May 2021

Published: 24 June 2021

Citation:

Feringa FM and van der Kant $R$ (2021) Cholesterol and Alzheimer's

Disease; From Risk Genes to Pathological Effects.

Front. Aging Neurosci. 13:690372. doi: 10.3389/fnagi.2021.690372

\section{Cholesterol and Alzheimer's Disease; From Risk Genes to Pathological Effects}

\author{
Femke M. Feringa ${ }^{1,2}$ and Rik van der Kant ${ }^{2,3 *}$ \\ ${ }^{1}$ Department of Clinical Genetics, Center for Neurogenomics and Cognitive Research (CNCR), Amsterdam University \\ Medical Center, Amsterdam, Netherlands, ${ }^{2}$ Department of Functional Genomics, Center for Neurogenomics and Cognitive \\ Research (CNCR), VU University Amsterdam, Amsterdam, Netherlands, ${ }^{3}$ Alzheimer Center Amsterdam, Department of \\ Neurology, Amsterdam Neuroscience, Amsterdam University Medical Center, Amsterdam, Netherlands
}

While the central nervous system compromises $2 \%$ of our body weight, it harbors up to $25 \%$ of the body's cholesterol. Cholesterol levels in the brain are tightly regulated for physiological brain function, but mounting evidence indicates that excessive cholesterol accumulates in Alzheimer's disease (AD), where it may drive AD-associated pathological changes. This seems especially relevant for late-onset AD, as several of the major genetic risk factors are functionally associated with cholesterol metabolism. In this review we discuss the different systems that maintain brain cholesterol metabolism in the healthy brain, and how dysregulation of these processes can lead, or contribute to, Alzheimer's disease. We will also discuss how AD-risk genes might impact cholesterol metabolism and downstream AD pathology. Finally, we will address the major outstanding questions in the field and how recent technical advances in CRISPR/Cas9gene editing and induced pluripotent stem cell (iPSC)-technology can aid to study these problems.

Keywords: cholesterol, Alzheimer, ApoE, gliosis, iPSC models

\section{INTRODUCTION}

Dementia affects over 46 million people worldwide, a number that is expected to double within the next 20 years due to our increased life expectancy (Prince et al., 2015). Alzheimer's Disease $(\mathrm{AD})$ is the most common type of dementia and no successful treatment that can cure AD, or halt its progression, is available today. At the pathological level, $\mathrm{AD}$ is characterized by the accumulation of extracellular amyloid beta $(\mathrm{A} \beta)$ plaques, and intracellular neurofibrillary tangles (NFT) consisting of hyperphosphorylated Tau species (Scheltens et al., 2016). AD can develop early ( $<65$ years) referred to as early-onset $\mathrm{AD}$ (EOAD), which is in part explained by autosomal dominant inheritance of coding mutations in the amyloid precursor protein (APP) or presenilin genes (PSEN1 and PSEN2), in that case called familial AD (FAD). The FAD related mutations directly affect $A \beta$ production and their identification therefore contributed to formation of the amyloid cascade hypothesis that postulates a linear relation between development of $A \beta$ plaques and NFT in AD (Pimenova et al., 2018). Whilst EOAD only represents around 5\% of all AD cases, the vast majority of $\mathrm{AD}$ patients suffer from late-onset $\mathrm{AD}$ (LOAD), for which aging is the biggest risk factor in addition to genetic and lifestyle factors (Scheltens et al., 2016). Multiple studies on the lifestyle and genetic interactions with $\mathrm{AD}$ have connected altered circulating cholesterol metabolism and hypercholesterolemia with aging and $\mathrm{AD}$ pathogenesis (Box 1). 
BOX 1 | Hypercholesterolemia, high fat diet and AD.

With the identification of lipoprotein ApoE4 as the biggest risk factor for LOAD in the early nineties, the interest for lipids and cholesterol metabolism in AD rapidly developed (Corder et al., 1993; Saunders et al., 1993; Strittmatter et al., 1993). Expression of the ApoE4 allelic variant of ApoE had previously been shown to increase plasma low-density lipoprotein (LDL) levels and increase the risk for atherosclerosis. Moreover, carriers of the ApoE4 gene were overrepresented in hyperlipidemic and cardiovascular patients (Huang, 2010; Mahley, 2016). At the epidemiological level, retrospective studies have shown that obesity, type 2 diabetes, cardiovascular disease and hypercholesterolemia at middle-age increase the risk for dementia at older age in humans (Pappolla et al., 2003; Whitmer et al. 2005; Stampfer, 2006; Solomon et al., 2009; Pedditizi et al., 2016; Anstey et al., 2017; Ma et al., 2020; Tini et al., 2020; Barbiellini Amidei et al., 2021). In line, increased plasma and CSF levels of the cholesterol metabolite 24-hydroxycholesterol (24S-OHC) that is selectively generated in neurons, have been linked to early AD development (Lütjohann et al., 2000; Papassotiropoulos et al., 2002; Schönknecht et al., 2002; Li et al., 2018). Cholesterol has also been shown to accumulate in mature A $\beta$-plaques in AD patients and APP(SW) mice (Mori et al., 2001) and cholesterol levels in the brain positively correlate with the severity of dementia in AD patients (Cutler et al., 2004). In line, lower AD incidence was associated with statin use in retrospective studies (Jick et al., 2000; Wolozin et al., 2000; Cramer et al., 2008; Haag et al., 2009; Li et al., 2010; Lin et al., 2015; Zissimopoulos et al., 2017; Zhang et al., 2018). The protective effect of statin usage was present independent of ApoE status (Haag et al., 2009; Li et al., 2010). A subset of these studies showed that the protective effect of statin usage was no longer present in participants that fell within the oldest age categories (>80). This could be due to a survival bias, where participants that survive till old age have fewer additional medical conditions, that would have increased their risk for AD development. Alternatively, this could point to a beneficial effect of statin usage only when taken at a timepoint before pathological hallmarks of $A D$ would typically develop in the brain. A link between high circulating cholesterol levels and $A D$ was also corroborated in AD mouse models where a hypercholesterolemic diet increased A $\beta$-plaque load (Refolo et al., 2000). In addition, high cholesterol diet in mice induced Tau hyperphosphorylation, which was amplified by loss of ApoE expression (Rahman et al., 2005; Glöckner et al., 2011). Glial activation, contributing to gliosis as seen in $A D$, has also been reported in mice on a high cholesterol diet (Crisby et al., 2004). Metabolic changes accompanied by AD phenotypes in the brain, where also described in rabbits on a high cholesterol diet (Jin et al., 2018). Finally, a high fat/high cholesterol diet in young (4-month old) versus aged (14-month old) rats negatively affected memory performance at both ages, while also increasing hippocampal pTau levels at old age, indicating the detrimental combination of disturbed circulating cholesterol homeostasis and aging (Ledreux et al., 2016).

In the brain, already a century ago, in addition to plaques and tangles, Dr. Alzheimer described as a third characteristic of AD: the extensive accumulation of 'adipose saccules' (Alzheimer, 1907). These 'adipose saccules' were likely what we now refer to as lipid droplets, and are major storage organelles of intracellular lipids such as cholesterol and fatty acids (Farmer et al., 2020). While mostly ignored since their first discovery, these 'adipose saccules' in the brain have gained renewed interest in light of the findings on cholesterol metabolism and $\mathrm{AD}$ in the last two decades.

In this review, we will discuss the basic regulation of cholesterol homeostasis at the cellular level, and how crosstalk between different brain-cell types regulates "healthy" cholesterol homeostasis in the human brain. We will then discuss how brain cholesterol metabolism is affected by aging and how neuronal cholesterol can contribute to downstream AD pathologies such as Amyloid- and Tau accumulation. Next, we will examine the contribution of human-specific $\mathrm{AD}$ risk polymorphisms to cholesterol dyshomeostasis and gliosis in different brain cell types, an area of research that greatly benefits from the development of CRISPR gene-editing and iPSC techniques. Lastly, we will formulate some of the major questions still outstanding in the field, and how they could be addressed to develop disease-modifying interventions for $\mathrm{AD}$ based on our knowledge of cholesterol metabolism in AD.

\section{INTRACELLULAR CHOLESTEROL METABOLISM; THE BASICS}

The largely hydrophobic molecule cholesterol localizes primarily in cell membranes where it regulates membrane fluidity and can interact with neighboring lipids and proteins to regulate membrane trafficking or signal transduction (Luo et al., 2019). Cholesterol levels in cells are balanced by de novo biosynthesis, uptake, storage and export [extensively reviewed by
Luo et al. (2019)]. In short: De novo synthesis of cholesterol starts when sterol regulatory element binding protein 2 (SREBP2), the key regulator of cholesterol synthesis, transfers from the endoplasmic reticulum (ER)-membrane to the Golgi where it is processed in order to enter the nucleus and induce transcription of its numerous target genes involved in cholesterol synthesis (Figure 1). Together around 30 consecutive reactions ensure cholesterol synthesis in the ER starting from acetyl-CoA. HMG-CoA reductase (HMGCR) and squalene monooxygenase (SM), both SREBP2 targets, are rate limiting enzymes in this process. Cholesterol is transported from the ER to the plasma membrane without passing through the Golgi (Dai et al., 2021). As an alternative to synthesis, cells can acquire cholesterol trough uptake. When not incorporated in the lipid bilayer of a membrane, most cholesterol is protein bound in apolipoprotein particles that facilitate transport (Zhang and Liu, 2015). Uptake of these cholesterol containing particles depends on Low-density lipoprotein receptors (LDLRs) on the plasma membrane. Binding of lipoprotein particles to the LDLR causes incorporation of LDL into clathrin-coated vesicles that enter the endocytic pathway (Goldstein and Brown, 2009). LDLR is either recycled via endosomal recycling or directed to lysosomes for degradation (Rudenko et al., 2002; Bartuzi et al., 2016; Fedoseienko et al., 2018). When LDLs arrive in lysosomes, cholesterol is freed by hydrolysis of CEs present in the LDLs. NPC2, NPC1 and lysosome-associated glycoprotein LAMP2 control delivery of the newly formed cholesterol to the ER (Kwon et al., 2009). Excess cholesterol can be stored in lipid droplets as CEs and converted back to cholesterol by acidic lipases in the lysosome when needed (Ikonen, 2008). As an alternative to intracellular storage, excess cholesterol can also be exported as part of LDLor High-density lipoprotein (HDL) particles in a process named reverse cholesterol transport. This is mediated through ATPbinding cassette $(\mathrm{ABC})$ transporters, like $\mathrm{ABC}$ subfamily $\mathrm{A}$ member 1 (ABCA1) and ABC subfamily G member 1 (ABCG1), which are widely expressed in the body and coordinately 


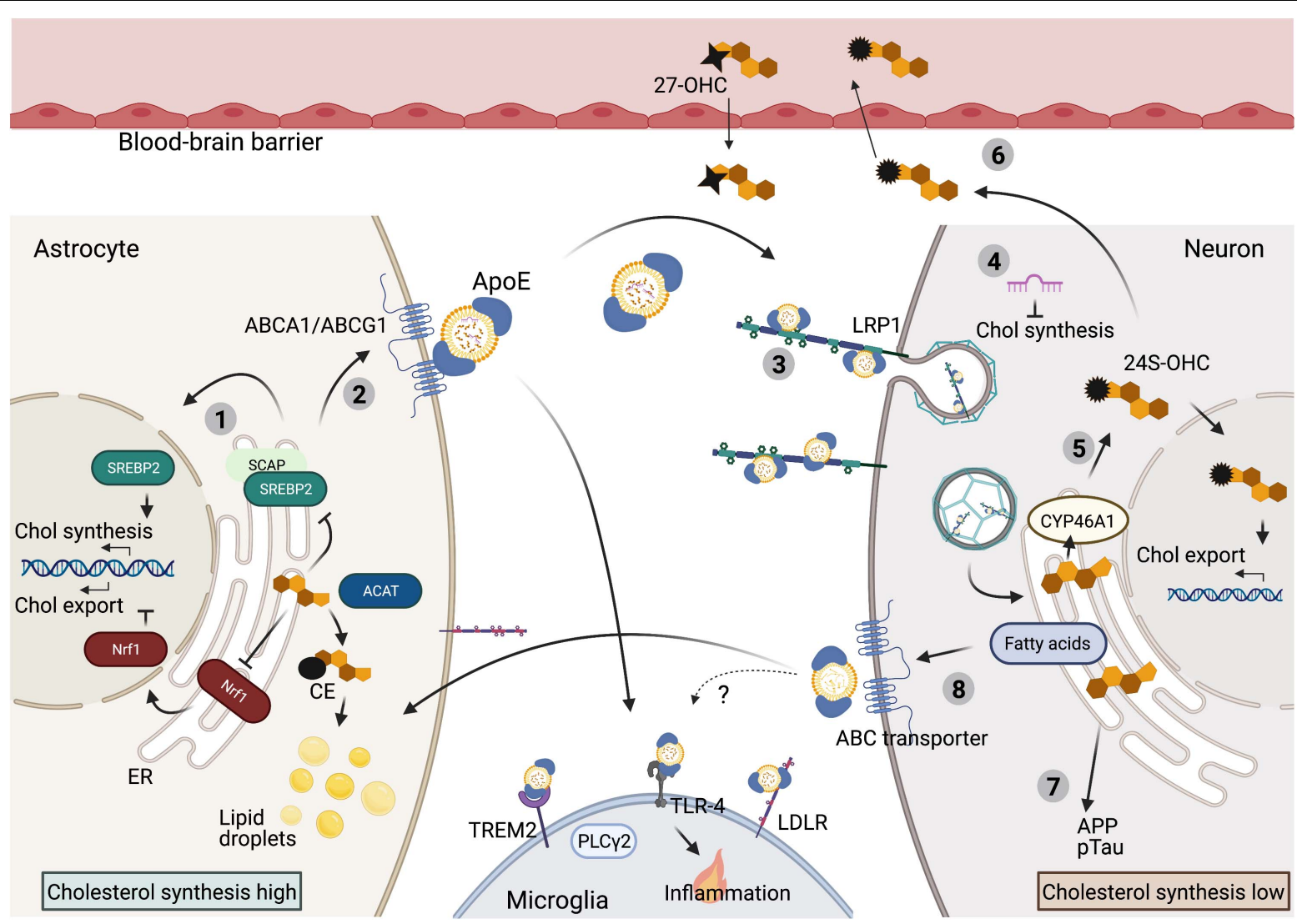

FIGURE 1 | Cholesterol metabolism in the brain. Overview of cholesterol metabolism in the mature brain. (1) In the brain cholesterol is predominantly synthesized in astrocytes. Cholesterol synthesis is under tight control of ER-cholesterol level. High ER-cholesterol concentrations prevent SREBP2 processing and thereby suppress cholesterol synthesis. High ER-cholesterol levels also inhibit Nrf1 processing to induce cholesterol export. In addition, excess ER cholesterol is converted to CE for storage in lipid droplets. (2) Cholesterol and cholesterol precursors are exported via ABC transporters and transported from astrocytes to neurons and microglia via ApoE bound lipoprotein-particles. (3) These lipoprotein particles can bind to lipoprotein receptors (LRP1 on neurons, TREM2, TLR-4 and LDLR on microglia) to be internalized. (4) Neuronal cholesterol synthesis is inhibited by ApoE dependent delivery of astrocytic derived microRNAs that target cholesterol synthesis genes in neurons. (5) Specifically in neurons, excess cholesterol is converted to $24 \mathrm{~S}-\mathrm{OHC}$, which activates a transcriptional program to promote cholesterol export. (6) 24S-OHC itself can be secreted from the brain via crossing of the BBB, while 27OHC can enter the brain from the periphery. (7) High cholesterol load in neurons can contribute to amyloidogenic APP processing and pTau accumulation. (8) Astrocytes can also prevent toxic overload of (peroxidized) fatty acids in neurons via ApoE-dependent lipid-particle transport from neurons to astrocytes, but whether cholesterol is also transported into this direction remains unknown (BioRender, 2021).

regulate cholesterol export from the cell (Figure 1) (Luo et al., 2019). Although the exact mechanism is still under debate, cholesterol effluxed by ABCA1 appears to be loaded on lipid-free Apolipoprotein A-I (ApoA-I), which can subsequently acquire additional cholesterol from ABCG1 (Gelissen et al., 2006).

While representing only $1 \%$ of cellular cholesterol, cholesterol levels in the ER play a central role in the regulation of all aspects of cholesterol metabolism described above. When cholesterol levels are low, SREBP2 interacts with SCAP in the ER membrane which promotes SREBP2 trafficking to the Golgi, it's processing and transcription of cholesterol-synthetic genes (Sakai et al., 1997, 1998; Brown et al., 2018). The uptake of cholesterol is also directly regulated through this process, as LDLR is a transcriptional target of SREBP2 (Luo et al., 2019). In this way, low ER cholesterol drives increased synthesis and uptake of cholesterol in order to balance cellular cholesterol levels (Figure 1). Reversely, too high levels of cholesterol can be toxic to cells. Therefore, when a surplus of cholesterol accumulates in the plasma membrane, cholesterol is transported back to the ER where it (i) inhibits SREBP2 activation and (ii) can be esterified by acyl-coA: cholesterol acyltransferase (ACAT1) to from CE for storage in lipid droplets (Chang et al., 1997; Zhang and Liu, 2017). Furthermore, when cholesterol levels in the ER are high, cholesterol is converted to oxysterols (Olsen et al., 2012). As the major sensor for cholesterol overload in a cell oxysterols prevent SREBP2 activation and directly activate the Liver $\times$ receptor (LXR), which promotes cholesterol efflux by transcription of ABC transporters (Radhakrishnan et al., 2007; Olsen et al., 2012). In addition to SREBP2, the transcription factor Nuclear factor erythroid 2-related factor 1 (NFE2L1 aka Nrf1) also senses ER cholesterol levels. When ER cholesterol levels are low, Nrf1 is cleaved and the transcription-part domain enters the nucleus where it inhibits LXR dependent transcription and thus prevents cholesterol export. When ER cholesterol levels rise, cholesterol 
binding to Nrf1 prevents its translocation to the nucleus, which causes de-repression of the LXR locus and promotes cholesterol efflux (Figure 1) (Widenmaier et al., 2017). Together, in a YinYang manner, SREBP2 and Nrf1 sense ER cholesterol levels to maintain cellular cholesterol homeostasis (Figure 1).

It is important to note, that many basic rules underlying intracellular cholesterol metabolism have been uncovered so far, as discussed above. However, most of this knowledge is acquired from experiments in dividing fibroblast culture systems. Cholesterol metabolism in the central nervous system (CNS) and, particularly in neurons, is under added pressure due to the postmitotic nature of these cells, their long-life span, large size and highly specialized metabolic demand which requests specific mechanisms to maintain lifelong cholesterol homeostasis in the brain.

\section{CHOLESTEROL METABOLISM IN THE BRAIN; DIFFERENT NEEDS FOR DIFFERENT CELLS}

Due to the blood-brain barrier (BBB) cholesterol metabolism in the CNS is largely separated from the periphery and it is generally understood that little diet-derived cholesterol enters the brain (Björkhem and Meaney, 2004). Therefore the brain is largely dependent on its own cholesterol synthesis and separate metabolism regulated by a complex interplay between different highly specialized cell types, each with their own demand for cholesterol (Dietschy and Turley, 2004). In adults, biosynthesis of cholesterol is thought to almost exclusively take place in astrocytes, from where cholesterol is transported to neurons via ApoE lipoproteins (Figure 1) (Pfrieger and Ungerer, 2011). Although cell type specific cholesterol synthesis rates have not been determined in vivo, cholesterol synthesis rates in cultured rat astrocytes are double as high as in cultured neurons (Nieweg et al., 2009). Moreover, conditional depletion of cholesterol synthesis in neuronal cells in mice did not result in neurodegeneration or synapse loss, indicating that mature neurons can acquire sufficient cholesterol levels supplied by surrounding glia (Fünfschilling et al., 2007). Accordingly, neuronal synaptogenesis has been shown to depend on ApoE dependent cholesterol transport from astrocytes to neurons (Mauch et al., 2001; Pfrieger, 2003). Of interest is the recent finding that astrocytes might also suppress cholesterol synthesis in neurons, as astrocytic ApoE was shown to deliver microRNAs to neurons that target and suppress expression of cholesterol biosynthesis genes (Li et al., 2021). Astrocyte-derived lipoproteins carry cholesterol and phospholipids as well as cholesterol precursors, presumably used by neurons for processing, but contain little $\mathrm{CE}$ or triglycerides making them substantially different from plasma lipoproteins (Pfrieger and Ungerer, 2011). Instead of ApoA-I, ApoE is the main apolipoprotein responsible for lipid transport in the CNS. ApoE is highly expressed in astrocytes where it is lipidated and exported via $\mathrm{ABC}$ transporters like ABCA1 and ABCG1 (Figure 1) (Koldamova et al., 2003; $\mathrm{Xu}$ et al., 2006). Which ABC transporters are responsible for cholesterol efflux in the CNS seems to be cell-type dependent.
Blocking ABCA1 or ABCG1 mediated transport in primary rat astrocytes reduced ApoE mediated cholesterol export, but had no effect on cholesterol efflux from primary cultured neurons. In contrast, knock down of ABCG4 selectively affected cholesterol export in primary cultured neurons (Chen et al., 2013). Neurons can take up astrocyte-derived HDL-like lipoprotein particles containing ApoE through receptors of the LDLR family (Figure 1), of which LRP1 is highest expressed in neurons (Vance and Hayashi, 2010). Similar to neurons, cholesterol biosynthesis levels are relatively low in microglia, which also mainly depend on astrocytes for cholesterol production (Zhang et al., 2014; Loving and Bruce, 2020). On the microglial cell surface, ApoE lipoprotein particles can interact with Triggering Receptor Expressed on Myeloid Cells 2 (TREM2), Toll Like Receptor 4 (TLR-4) and the LDLR to internalize lipoprotein particles into the microglia (Figure 1) (Loving and Bruce, 2020).

As mentioned above, regulation of cholesterol metabolism is particularly important for neurons. To further fine-tune cholesterol metabolism, neurons contain another cholesterolregulating enzyme; cholesterol 24-hydroxylase (CYP46A1), which is CNS specific and under healthy conditions only expressed by neurons (Brown et al., 2004; Ramirez et al., 2008). CYP46A1 converts excess cholesterol to 24S-hydroxycholesterol (24S-OHC) (Lund et al., 2003; Ramirez et al., 2008; Zhang and Liu, 2015; van der Kant et al., 2019), which can be released by neurons and crosses the $\mathrm{BBB}$ through diffusion, forming a major export pathway for excess cholesterol from the brain (Figure 1) (Lütjohann et al., 1996; Lund et al., 1999, 2003; Xie et al., 2003). Due to its neuron specific origin, 24S-OHC levels in the blood also provide a direct measure of cholesterol turnover levels in the brain (Sodero, 2020). Besides being an export product, as other oxysterols, 24S-OHC can promote ApoE-mediated cholesterol export by activating liver $\mathrm{X}$ receptor (LXR) (Figure 1) (Abildayeva et al., 2006; Matsuda et al., 2013). Additional oxysterols that are produced in the brain include 27-OHC, which is generated by the enzyme CYP27A1 and can be further processed by CYP7B to form $7 \alpha$-hydroxy-3-oxo-4cholestenoic acid (7-OH-4-C). 7-OH-4-C can cross the BBB to be eliminated by the liver. CYP27A1 is expressed in multiple brain cell types, yet 27-OHC levels in the brain are only a fraction of the far more abundant 24S-OHC (Brown et al., 2004; Heverin et al., 2004; Gilardi et al., 2009). In fact most 27-OHC is not produced in the brain but enters the brain via the $\mathrm{BBB}$ originating outside the CNS (Gamba et al., 2015).

While neurons depend on the astrocyte-to-neuron lipid shuttle for supply of cholesterol and cholesterol precursors, recent studies have shown that lipids under certain circumstances can also be transported from neurons to astrocytes. For example, neuronal lipids can become peroxidized when they encounter oxidative stress, potentially generated due to hyperactivity or as an incidental of aging. Neurons are not well equipped to deal with these toxic lipids, and peroxidized lipids in neurons are therefore transported to astrocytes in an ApoE-dependent manner (Figure 1) (Liu et al., 2015, 2017; Moulton et al., 2021; Qi et al., 2021; Ioannou et al., 2019). Astrocytes store these lipids while also upregulating expression of genes responsible for oxidative energy metabolism to process these peroxidized lipids, 
thereby protecting neuronal integrity (Ioannou et al., 2019). While this directional of lipid-transport is now well established for fatty acids, it is unknown how this neuron-to-astrocyte lipid shuttle affects cholesterol transport and overall cholesterol metabolism in the healthy brain.

\section{BRAIN CHOLESTEROL METABOLISM; CHANGES FROM DEVELOPMENT TO AGING}

The processes that maintain cholesterol homeostasis in the healthy brain are not static, but change during early human development and later again in the aging brain. Cholesterol synthesis rates are at the highest-level during brain development to support the generation of an extensive neuronal network (Waelsch et al., 1940; Quan et al., 2003; Pfrieger and Ungerer, 2011). Therefore, all brain cell types, including both neurons and glial cells are thought to contribute to cholesterol biosynthesis during development (Pfrieger and Ungerer, 2011; Genaro-Mattos et al., 2019). Once an adult, brain cholesterol synthesis rate declines and astrocytic cholesterol production ensures sufficient levels to support neuronal plasticity and glial performance (Andersson et al., 1990; Lütjohann et al., 1996; Dietschy and Turley, 2004; Zhang and Liu, 2015). A further decline in cholesterol synthesis upon aging is suggested by detection of lower cholesterol precursor levels in post mortem hippocampal tissue from middle-aged and elderly ( $>38$ years) donors compared to young ( $<38$ years) donors (Thelen et al., 2006). Yet, absolute cholesterol levels were stable in aged human hippocampal tissue, while a decrease is observed in white and gray matter regions (Söderberg et al., 1990; Thelen et al., 2006). Hippocampal 24S-OHC levels showed a downward trend in middle-aged and elderly ( $>38$ years) donors, which also suggests a decrease in cholesterol metabolism and cholesterol turnover (Thelen et al., 2006). Possibly, lower cholesterol turnover helps to keep cholesterol levels relatively stable in the aging brain when cholesterol synthesis decreases, but less cholesterol turnover might also contribute to reduced neuronal plasticity associated with aging (Thelen et al., 2006). In addition, the BBB, which normally separates CNS and peripheral cholesterol, has been shown to lose integrity during aging (Montagne et al., 2015). This might affect brain cholesterol levels especially in the hippocampus where BBB break-down has been reported to occur first (Montagne et al., 2015; Segarra et al., 2021). Indeed in mice, $\mathrm{BBB}$ breakdown results in entry of peripheral cholesterol into the brain, and reversely $\mathrm{BBB}$ breakdown also led to increased release of $24 \mathrm{~S}-\mathrm{OHC}$ from the brain into the circulation (Saeed et al., 2014). Brain cholesterol synthesis was increased upon BBB disruption in mice, which might be induced to compensate for the lowered 24S-OHC level (Saeed et al., 2014). What happens to intracellular levels of cholesterol in neurons, astrocytes and microglia during the aging process or downstream of $\mathrm{BBB}$ breakdown is not well known.

Age-associated neurodegeneration itself also has a major impact on brain cholesterol metabolism. For example, dying neurons generate high levels of cholesterol-rich debris, in part due to the dismantling of myelin sheets formed by oligodendrocytes. This debris is subsequently phagocytosed by microglia (Callaghan et al., 2014). Recently, CantutiCastelvetri et al. (2018) showed that in the aged brain, phagocytes (mainly representing microglia) had lost the ability to process excess amounts of cholesterol, which depended on ApoE and led to accumulation of cholesterol into crystals in the phagocytic cells. The intracellular accumulation of cholesterol in microglia induced an inflammatory response and prevented successful re-myelination. Re-myelination could be restored by stimulation of reverse cholesterol transport or inhibition of the inflammatory response, indicating that aging can affect the cholesterol efflux capacity of immune cells in the brain, which perturbs timely reversal of immune responses needed for proper re-myelination (Cantuti-Castelvetri et al., 2018). Increased presence of lipid droplets, which are storage sites for neutral lipids like glycerolipids and CEs has also been observed in aged microglia of both mouse and human brains (Shimabukuro et al., 2016; Farmer et al., 2020; Marschallinger et al., 2020). While the lipid composition in these droplets has not been well characterized in humans, lipidomic analysis was performed on lipid-droplet containing microglia from aged mouse hippocampus. These lipid droplets contained predominantly glycerolipids, like triacylglycerols (TAGs), diacylglycerols (DAGs), and monoacylglycerols (MAGs), but little $\mathrm{CE}$, indicating that cholesterol is not a major contributor to this age-related phenotype at least in mice (Marschallinger et al., 2020). Therefore, these so-called Lipid-droplet-accumulating microglia (LDAM) seem to be distinct from the microglia in aged mice that accumulated cholesterol after de-myelination (CantutiCastelvetri et al., 2018; Marschallinger et al., 2020). In contrast to the general aging process, in $\mathrm{AD}$ intracellular cholesterol accumulation has been broadly reported for a number of cell types, as discussed below.

\section{CHOLESTEROL AND AD, A DUAL DRIVER OF A $\beta$ AND TAU PATHOLOGY IN NEURONS}

\section{Cholesterol, APP Processing and A $\beta$ Generation in Neurons}

The relationship between cholesterol metabolism, APP processing and $A \beta$ production has been characterized in much detail. $A \beta$ is generated when the amyloid precursor protein (APP) is sequentially processed by $\beta$-secretase (BACE1) and $\gamma$-secretase. Alternatively, APP can be cleaved by $\alpha$-secretases, a pathway known as the non-amyloidogenic pathway. APP is normally present in the bilayer membrane of the cell and concentrated in neuronal synapses (Zheng and Koo, 2011). Exogenous addition of cholesterol to human brain tissue lysates promoted BACE1 and $\gamma$-secretase activity (Xiong et al., 2008) and treatment of primary mouse neuronal cultures with excess cholesterol was sufficient to increase $A \beta_{42}$ secretion (Marquer et al., 2014). In addition, exogenous cholesterol addition in APP transfected HEK293 cells reduced APP processing via the non-amyloidogenic pathway 


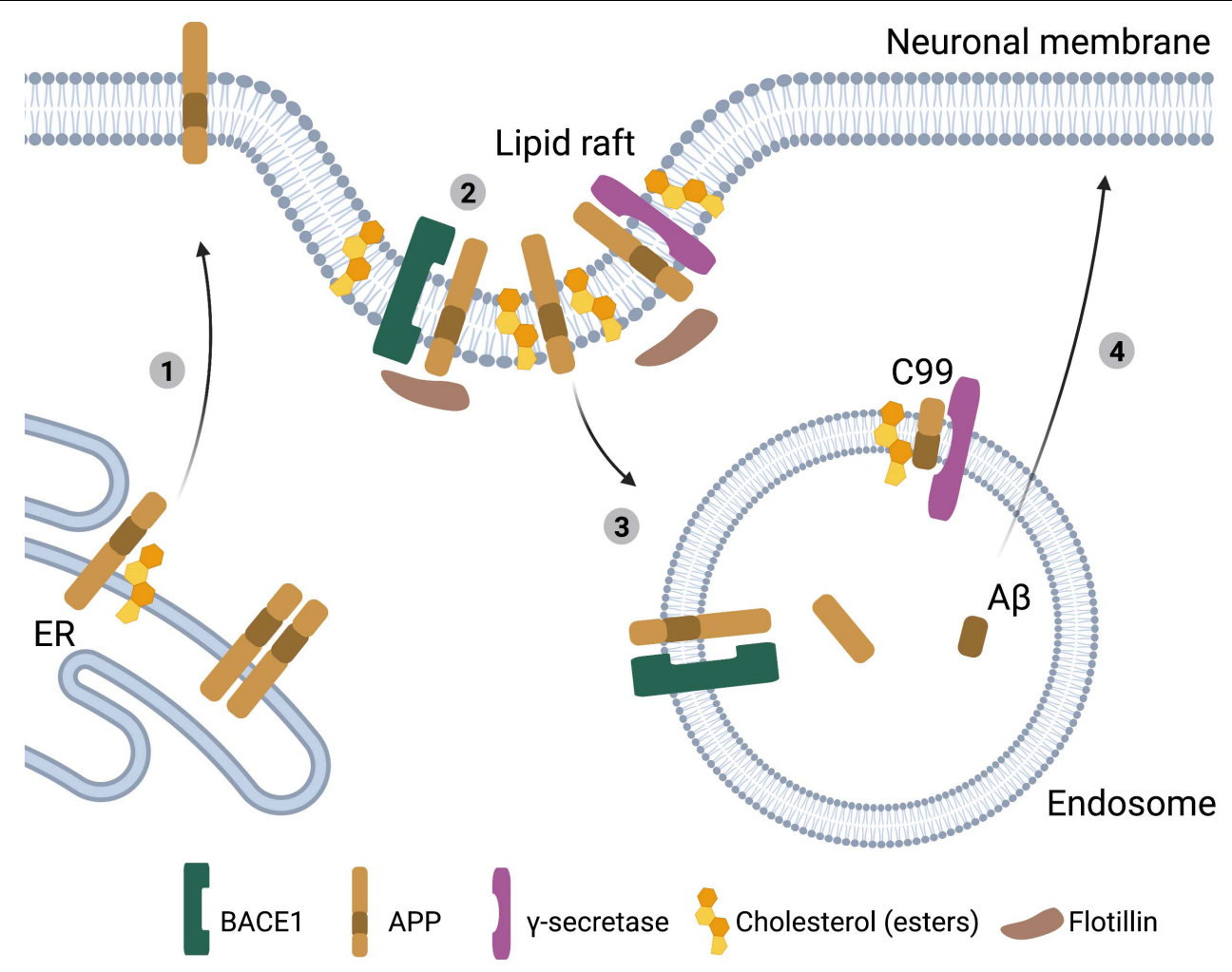

FIGURE 2 | Cholesterol, APP processing and A generation in neurons. Schematic representation of the interactions between cholesterol and APP processing in neurons. (1) High ER-cholesterol levels prevent APP dimerization and promote APP transport to the plasma membrane. (2) Cholesterol or cholesterol esters in the plasma membrane stimulate the clustering of APP, BACE1 and $\gamma$-secretases in lipid rafts. (3) These lipid rafts undergo clathrin-mediated endocytosis resulting in amyloidogenic processing of APP to A $\beta$. (4) A $\beta$ peptides are subsequently secreted from the neuron (BioRender, 2021).

by $\alpha$-secretase (Bodovitz and Klein, 1996). Accordingly, lower cholesterol levels have been shown to inhibit APP processing by BACE1 and $\gamma$-secretase, while promoting processing of APP via the non-amyloidogenic pathway (Simons et al., 1998; Kojro et al., 2001; Refolo et al., 2001; Schneider et al., 2006; Grimm et al., 2008). These effects of cholesterol on APP processing could be mediated by effects of cholesterol on membrane composition. As a transmembrane protein APP can localize in lipid rafts, which are small sterol- and sphingolipid enriched domains that facilitate protein and lipid interactions and play a role in cellular signaling and membrane transport (Hicks et al., 2012). Multiple studies together uncovered that an increase in cholesterol levels promotes APP and BACE1 colocalization in lipid rafts which promotes clathrin-mediated endocytosis and APP processing via the amyloidogenic pathway (Figure 2) (Wahrle et al., 2002; Cordy et al., 2003; Ehehalt et al., 2003; Osenkowski et al., 2008; Cossec et al., 2010; Marquer et al., 2011). This is supported by a recent study in human iPSC-derived neurons, where lowering cholesterol levels reduced the interaction between full-length APP (flAPP) and BACE1, potentially explaining why APP processing is inhibited and flAPP levels are increased upon statin treatment (Langness et al., 2021). In addition, a cholesterol dependent interaction between flotillin and APP in lipid rafts might further promote endocytosis (Chen et al., 2006; Schneider et al., 2008; Cho et al., 2020). Importantly the cholesterol effect is APP specific, as higher membrane cholesterol levels did not affect endocytosis of other membrane proteins like transferrin (Cossec et al., 2010). Also, high membrane cholesterol levels only promote endocytosis and amyloidogenic processing of APP that is localized in lipid rafts, while APP located in the membrane outside of lipid rafts is unaffected (Cho et al., 2020). When analyzing lipid raft composition in postmortem AD vs. control brain tissue, Fabelo et al. (2014) actually detected lower cholesterol, but higher CE presence in lipid rafts of AD subjects, indicating that plasma membrane $\mathrm{CE}$ might also contribute to regulation of APP processing. Increased levels of $\mathrm{CE}$, have also been observed both in human postmortem $\mathrm{AD}$ brain tissue and in mouse models of AD (Chan et al., 2012; Tajima et al., 2013; Yang et al., 2014).

Cholesterol can also bind directly to APP in the transmembrane C-terminal domain (C99) (Beel et al., 2008; Barrett et al., 2012; Song et al., 2013; Nierzwicki and Czub, 2015). In iPSC derived neurons mutating the cholesterolbinding domain in APP results in reduced APP processing and $A \beta$ production (van der Kant et al., 2019). In addition, inhibition of $A \beta$ secretion by cholesterol-lowering statin treatment depended on the cholesterol binding domain in APP (van der Kant et al., 2019). Export of APP from the ER and subsequent APP processing to A $\beta$ is also cholesterol and/or CE dependent (Puglielli et al., 2001; Hutter-Paier et al., 2004; 
Huttunen et al., 2009; van der Kant et al., 2019), and regulated by the cholesterol-binding domain in APP (Langness et al., 2021). APP dimerization inhibits exit of flAPP from the ER, and subsequent processing to $\mathrm{A} \beta$ (Kaden et al., 2008; Eggert et al., 2009, 2018; Decock et al., 2015; Langness et al., 2021). Interestingly, residues required for binding of APP to cholesterol overlap with residues required for APP dimerization (Song et al., 2013), and thus binding of cholesterol to APP might prevent APP dimerization thereby enhancing ER-export of monomeric APP (Nierzwicki and Czub, 2015; Langness et al., 2021). Whether the cholesterol-binding domain of APP also directly affects APP recruitment into lipid rafts, thereby providing another level of cholesterol-dependent regulation of APP-processing is currently unknown. Also, whether ER cholesterol, or ER CE's are the main driver of these processes needs to be further established. It is, however, interesting to note, that like SREBP processing, the regulation of APP processing is very much dependent on intracellular cholesterol levels, raising the possibility that APP is a regulator of intracellular cholesterol homeostasis, which is also supported by a number of publications (Box 2). Overall, there is evidently a strong correlation between cholesterol levels and $\mathrm{A} \beta$ generation, with increased levels of cholesterol and/or CE in neurons driving $A \beta$ generation (Figure 2).

\section{Cholesterol and Tau}

In addition to an established connection between cholesterol and APP processing, cholesterol metabolism was also recently found

BOX 2 | Tables turned; APP as a regulator of cellular cholesterol metabolism. While cholesterol levels are known to regulate APP processing (Figure 2), accumulating data indicate that APP and its cleavage products can also regulate cholesterol metabolism in turn. In primary cultures of rat cortical neurons, higher expression of full length APP decreased HMGCR-mediated cholesterol synthesis, while lowering APP levels increased cholesterol biosynthesis (Pierrot et al., 2013). Similarly, deletion of APP caused increased SREBP2 target gene expression in human iPSC derived astrocytes (Fong et al., 2018). In addition, the C99 APP-fragment has been demonstrated to cluster cholesterol in the ER membrane thereby lowering de novo cholesterol synthesis (Montesinos et al., 2020), while the APP intracellular domain (AICD), a cytosolic fragment generated from C99, can directly bind to, and suppress, the LRP1 promoter thereby potentially lowering LRP1 dependent uptake of ApoE delivered cholesterol into neurons (Liu et al., 2007). In line with the position of the cholesterol binding domain in APP (Barrett et al., 2012), A $\beta_{40}$ and $A \beta_{42}$ peptides have been shown to bind extracellular cholesterol, thereby competing with ApoE or LDL driven cholesterol import, and reducing ApoE-dependent cholesterol delivery (Yao and Papadopoulos, 2002). In astrocytes, exogenous $A \beta$ stimulated cholesterol transport from plasma membrane to the Golgi, thereby lowering plasma membrane cholesterol levels (lgbavboa et al., 2009). Finally, $A \beta_{42}$ has been shown to inhibit astrocytic ABCA1 expression (Canepa et al., 2011), which would reduce cholesterol secretion and transport to neurons. Together these results show that APP processing and cleavage fragments can directly affect brain cholesterol homeostasis. This raises the interesting question whether FAD-associated mutations that affect APP-processing also alter brain cholesterol metabolism, which could then further contribute to AD pathology in a cholesterol-dependent manner. Indeed, accumulation of CE has been demonstrated in multiple mouse models of FAD (Chan et al., 2012; Tajima et al., 2013; Yang et al., 2014), indicating that altered brain cholesterol metabolism could hurry pathogenesis also in FAD. to directly regulate phosphorylated Tau (pTau) levels in iPSCderived neurons. As identified by an unbiased high-throughput drug screen, drugs that reduced CE levels in iPSC-derived neurons from familial AD (FAD) patients, also decreased pTau levels (van der Kant et al., 2019). This reduction of pTau was mediated by an increase in proteasomal degradation of pTau, and independent on the effect of CE on APP processing and $\mathrm{A} \beta$ (van der Kant et al., 2019). Interestingly, genetically lowering cholesterol esterification in triple-transgenic AD mice (3xTg-AD) mice also lowered pathological Tau accumulation (Shibuya et al., 2015). In addition, in vivo, genetic inhibition of ApoE-mediated cholesterol transport from astrocytes to neurons also reduced neuronal pTau levels in mice (Wang et al., 2020). While the exact mechanism underlying cholesterol-dependent regulation of Tau needs to be further established, these findings do further implicate cholesterol as a central player in $\mathrm{AD}$ pathogenesis upstream of $A \beta$ and Tau pathology (Kant et al., 2019).

\section{DYSREGULATION OF BRAIN CHOLESTEROL IN AD; IT IS IN THE GENES}

The last decade has seen the discovery of numerous genetic risk factors for LOAD by genome-wide-association studies (GWAS) on LOAD patients vs. healthy controls (Lambert et al., 2013; Jansen et al., 2019; Kunkle et al., 2019; Bellenguez et al., 2020). A high number of the LOAD risk genes have roles in lipid homeostasis, which is best defined for ApoE.

\section{ApoE}

Three common allelic ApoE genetic variants exist in the human population; ApoE2, ApoE3 and ApoE4. ApoE3 is the most common isoform present homozygous in over $60 \%$ of the population and is considered the reference allele for LOAD risk (Jeong et al., 2019). ApoE4 is a strong risk factor for LOAD: carriers of one ApoE4 allele have a 3 to 4 -fold increased risk for LOAD, while homozygous ApoE4 carriers have an approximate 14-fold increased risk of developing LOAD compared to ApoE3 carriers (Liu et al., 2013). It has to be noted, however, that the penetrance of the ApoE4-risk allele varies in different ethnicities, possibly due to differences in ApoE expression levels (Griswold et al., 2021). In contrast to ApoE4, expression of the ApoE2 allele confers a decreased risk for LOAD and hence is considered protective (Corder et al., 1994; Reiman et al., 2020). Despite their strong effects on LOAD risk, the three ApoE isoforms only differ from each other by two amino acids (Chen et al., 2020). ApoE3 contains a Cys112 and Arg158, of which Cys112 is changed to Arg112 in ApoE4 and Arg158 is changed to Cys158 in the ApoE2 variant (Figure 3). As an apolipoprotein ApoE interacts with lipoproteins to execute its function as a cholesterol and lipid carrier. Via a receptor binding domain ApoE can interact with lipoprotein receptors to be internalized and deliver the cargo of lipids to cells. As described above, this ApoE-dependent route is crucial for transport of cholesterol and cholesterol precursors from astrocytes to neurons in the mature brain. 


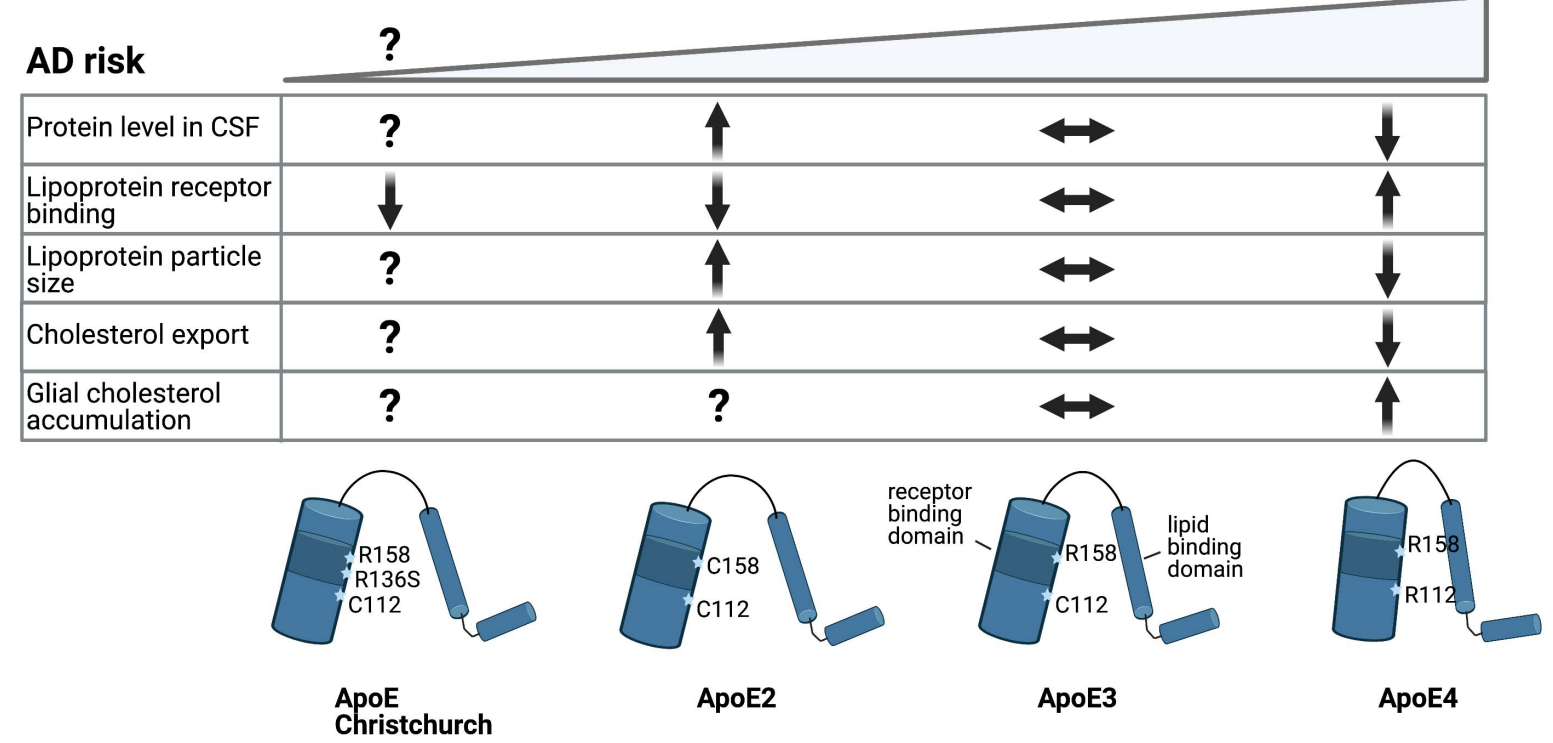

FIGURE 3 | Alzheimer's disease risk variants in ApoE affect cholesterol metabolism. Schematic representation of ApoE allelic variants and their consequences. Single amino acid changes in ApoE confer different risk for LOAD. Conformational change of the protein structure by expression of the different ApoE alleles changes LDL receptor binding and lipid particle binding capacity, which might affect brain cholesterol metabolism (BioRender, 2021).

Strikingly enough, while peripheral ApoE4 is a major risk factor for hypercholesterolemia (Box 1), it remains largely unknown how ApoE isoforms affect cholesterol metabolism in the brain. Astrocytes are the highest ApoE-expressing cell type in the brain (Huang et al., 2004), and transcriptomic analysis of human iPSC-derived neurons, astrocytes and microglia, revealed that ApoE4 driven changes in gene expression were most dramatic in astrocytes (Julia et al., 2019). Compared to ApoE3 astrocytes, ApoE4 astrocytes expressed higher levels of genes with a role in cholesterol biosynthesis and displayed cholesterol accumulation in lysosomes, while CE levels were not increased (Lin et al., 2018; Julia et al., 2019). The dysregulation in lipid metabolic genes in ApoE4 astrocytes was also confirmed in human control and AD brain samples (Julia et al., 2019). Also, an increased number of smaller lipid droplets has been detected in ApoE4 astrocytes compared to ApoE3 astrocytes derived from human ApoE-replacement mice (Farmer et al., 2019).

How ApoE4 affects cholesterol metabolism in other brain cell types like neurons and microglia is even less clear. ApoE4 expressing human iPSC-derived astrocytes showed reduced support of neuronal survival in an iPSC-derived neuronastrocyte co-culture compared to ApoE3 expressing astrocytes (Zhao et al., 2017). One way by which different ApoE polymorphisms could affect total brain lipid metabolism is by altering the export of ApoE-lipoprotein particles (Figure 3). In human CSF and upon overexpression in mice, ApoE2 has been shown to generate bigger HDL particles compared to ApoE3, while ApoE3 in turn is associated with bigger HDL particles then ApoE4, suggesting less sterol transport by ApoE4 (Hu et al., 2015; Heinsinger et al., 2016). Accordingly, an isoform dependent effect on cholesterol efflux, ApoE2 > ApoE3 > ApoE4, was detected previously in primary rat or mouse astrocytes and neurons (Michikawa et al., 2000; Rawat et al., 2019). No isoform dependent changes in binding of ApoE to ABCA1 were found that could explain reduced cholesterol efflux from ApoE4 expressing astrocytes, although ApoE4 has been suggested to affect ABCA1 membrane trafficking (Krimbou et al., 2004; Rawat et al., 2019). In addition, ABCA1 has recently been identified as a LOAD risk gene itself further implicating this pathway in LOAD pathogenesis (Bellenguez et al., 2020). In addition to export, ApoE genotype might also differentially affect the internalization of ApoE-lipoprotein particles. Lipidation of the ApoE protein triggers a conformational change that increases its binding affinity for the LDL receptors. Lipidated ApoE4 shows the strongest binding affinity for LDLR, while the binding of lipidated ApoE2 to LDLR is reduced compared to ApoE3 (Figure 3) (Chen et al., 2020). How these differences in receptor binding affinity affect uptake of ApoE-lipoprotein particles in specific brain cell types remains to be determined. The protein levels of ApoE also differ between isoforms, where ApoE2 levels are highest and ApoE4 levels are lowest in CSF and plasma (Castellano et al., 2011; Cruchaga et al., 2012). This might be a result of their different receptor affinities, as LDLR loss caused an increase in ApoE3 and ApoE4 levels, but not ApoE2 levels (Fryer et al., 2005). Importantly, the recently described Christchurch mutation in APOE (R136S) results in strongly reduced LDLR binding of ApoE, similar to ApoE2 (Figure 3). Presence of this mutation has been reported in an individual who had no signs of cognitive decline or Tau-pathology until advanced age despite carrying a PSEN1 mutation that causes autosomal-dominant AD (Arboleda-Velasquez et al., 2019). These results suggest a strong protective effect of the Christchurch ApoE variant, although this conclusion awaits further confirmation. Together, these results suggest strong ApoE genotype dependent effects 
on cholesterol metabolism in astrocytes. Whether, and how, this affects the proper astrocyte-dependent support of neuronal function and/or downstream $\mathrm{AD}$ pathology in neurons and glia remains to be determined.

Besides ApoE, other $\mathrm{AD}$ risk genes also have a predicted role in lipid metabolism. This is established for AD-genes that act in similar processes as ApoE and ABCA1, such as CLU and ABCA7 (discussed below), but also increasingly recognized for genes which are highly, or exclusively, expressed in microglia (discussed in the next section).

\section{CLU and ABCA7}

Like ApoE, clusterin (CLU) is a component of lipoproteins. The CLU gene producing clusterin (also referred to as ApoJ) is expressed in astrocytes and has a wide range of biological functions including cholesterol and lipid transport. In periphery, clusterin can form HDL particles that are transported to the liver (Baralla et al., 2015; Foster et al., 2019). Also, clusterin is expressed upon damage in arteries and could remove cholesterol from macrophage-foam cells that promote formation of atherosclerotic lesions (Gelissen et al., 1998; Foster et al., 2019). Clusterin plasma levels have been shown to correspond to severity of AD in patients (Thambisetty et al., 2010; Jongbloed et al., 2015), yet the specific role in brain cholesterol homeostasis is unknown.

Another AD risk gene, ABCA7 shares 54\% homology with ABCA1, the protein known to load cholesterol onto ApoE particles (Kaminski et al., 2000). However, what role ABCA7 has in intracellular cholesterol- and lipid transport remains unclear. ABCA7 is highly expressed in the brain, predominantly in neurons and microglia (Zhang et al., 2014). Reduced ABCA7 levels are observed in AD brain (Lyssenko and Praticò, 2020) and hippocampus of mice on a high fat diet (Zou et al., 2020). Contrary to ABCA1, transcription of ABCA7 is downregulated when cholesterol levels are high in the cell (Iwamoto et al., 2006). Recent studies on detergent purified ABCA7 showed that removal of cholesterol led to increased ATPase activity in ABCA7 (Le et al., 2021). In addition, ATPase activity was stimulated by interaction with apolipoproteins, ApoA-I and ApoE, where ATPase activity was hardly stimulated in presence of the ApoE4 compared to the ApoE3 variant. Interestingly, also the ApoE2 variant had a lower effect in stimulating ATPase activity of ABCA7 compared to ApoE3 (Le et al., 2021). The physiological consequences of these ApoE genotype dependent effects on ABCA7 and cholesterol metabolism remain to be determined. In vitro analysis in baby hamster kidney (BHK) cells transfected with either ABCA1-GFP or ABCA7-GFP revealed that free cholesterol efflux through ABCA7 was much lower compared to ABCA1 and was unaffected by ApoE genotype, while cholesterol efflux by ABCA1 was greatly reduced in presence of ApoE4 (Tomioka et al., 2017). Interestingly, recent data on the ABCA7 homolog in Drosophila suggests that ABCA7 might play a role in the neuron-to-glial transport of lipids to protect neuronal functionality and viability from toxic accumulation of peroxidized lipids, for instance generated by oxidative stress (Moulton et al., 2021). These results open the door for future studies on the role of ABCA7 in brain cholesterol metabolism and $\mathrm{AD}$ development.

\section{CHOLESTEROL AS A DRIVER OF (MICRO)GLIAL DYSFUNCTION AND GLIOSIS}

\section{TREM2, PLC $\gamma$ 2, and Microglial ApoE}

Multiple LOAD risk genes such as ApoE, TREM2 and PLC $\gamma 2$ are highly -or exclusively- expressed in (micro)glia and have been shown to regulate lipid metabolism and microgliosis. Increased expression of genes associated with lipid metabolism are found in microglia during development, damage or disease (Efthymiou and Goate, 2017; Keren-Shaul et al., 2017; Hammond et al., 2019; McQuade and Blurton-Jones, 2019). A good example is triggering receptor expressed on myeloid cells 2 (TREM2), which in brain is primarily expressed in microglia. Carriers of the $\mathrm{R} 47 \mathrm{H}$ or $\mathrm{R} 62 \mathrm{H}$ variant in this gene have an up to 4 -fold increased risk for LOAD (Ulrich and Holtzman, 2016). TREM2 has multiple ligands including the apolipoproteins ApoE and clusterin, and binding of TREM2 to these proteins is enhanced by their lipidation (Atagi et al., 2015; Bailey et al., 2015; Yeh et al., 2016). Binding of TREM2 to lipoprotein particles is reduced by the TREM2 R47H variant, which could lead to altered cholesterol load in microglia and also affects phagocytosis of lipoprotein bound $\mathrm{A} \beta$ by microglia (Yeh et al., 2016). Both astrocytes and microglia can phagocytose $A \beta$, thereby contributing to $\mathrm{A} \beta$ clearance from the brain (TarasoffConway et al., 2015; Ries and Sastre, 2016). Single-cell RNAsequence (RNA-seq) data from AD mouse models revealed socalled disease associated microglia (DAM) as a reactive microglial population that is generated when $\mathrm{AD}$ pathology is present in the brain (Keren-Shaul et al., 2017). The transition to a DAM-phenotype was dependent on TREM2 (Zhou et al., 2020). DAM have reduced expression of several homeostatic microglial genes accompanied by a significant increase in expression of lipid metabolism and phagocytosis genes, including ApoE. These activated DAM microglia localize predominantly around amyloid plaques where they form a neuroprotective barrier, prevent propagation of Tau pathology in mice and are believed to play a role in A $\beta$ clearance (Condello et al., 2015; Yuan et al., 2016; Keren-Shaul et al., 2017; Leyns et al., 2019). Of note, although increased ApoE levels were detected by recent (single-cell and single-nuclear) RNAseq studies in microglia from human AD postmortem brain tissue, a subpopulation with DAM signature was not identified (Grubman et al., 2019; Olah et al., 2020; Srinivasan et al., 2020; Zhou et al., 2020). While this may indicate differences between $\mathrm{AD}$ pathogenesis in mouse models and in humans, it could also be a consequence of technical limitations, e.g., too few single microglial cells analyzed or the low sensitivity of single-nuclear RNAseq to detect microglial activation genes (Del-Aguila et al., 2019; Mathys et al., 2019; Thrupp et al., 2020). As a consequence of TREM2 loss, microglia from TREM2 $2^{-/-}$mice on a demyelinating cuprizone (CPZ) diet failed to upregulate DAM-genes needed for cholesterol transport and lipid metabolism like ApoE, and could not clear myelin 


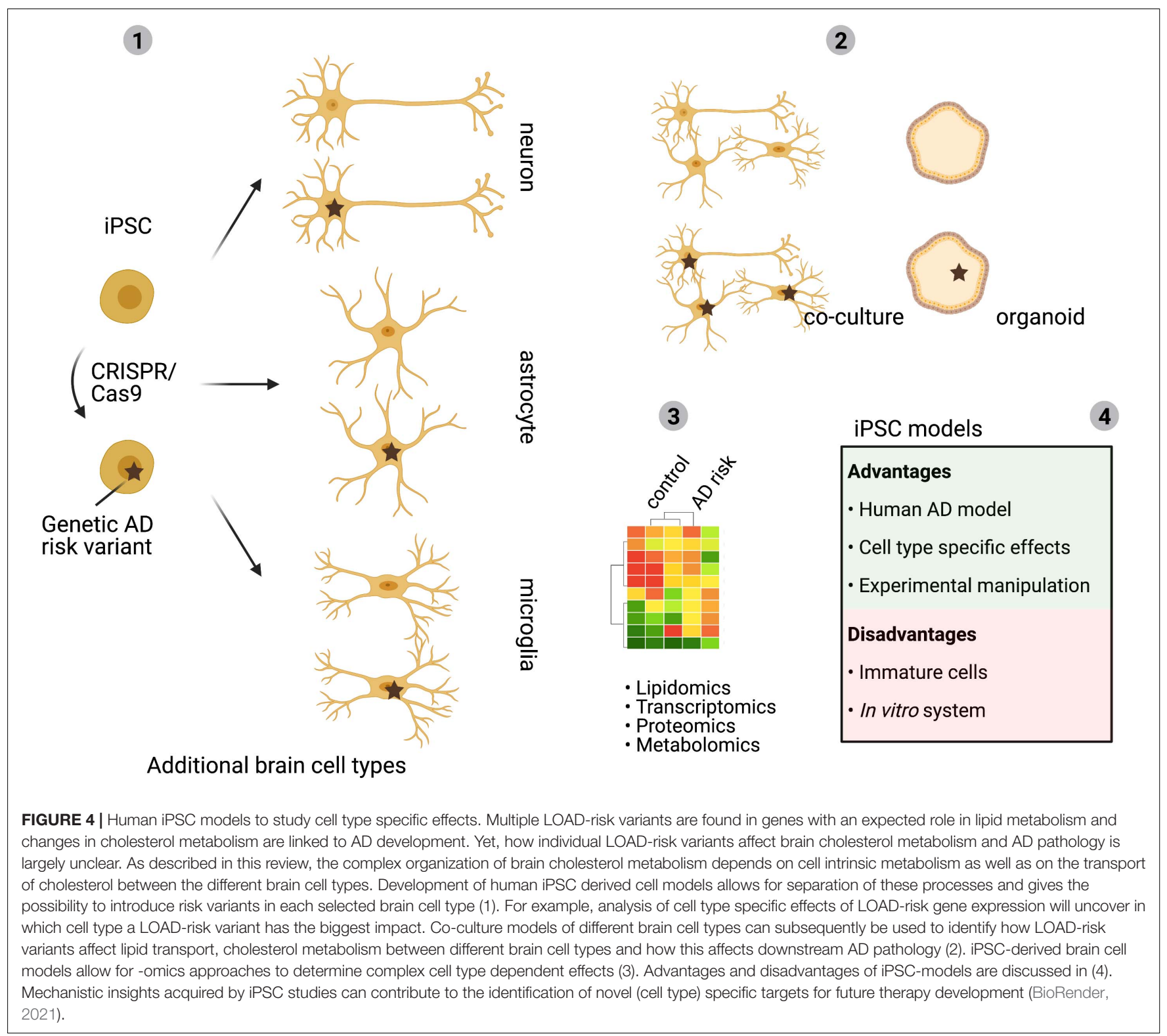

derived cholesterol, leading to microglial CE accumulation. A similar accumulation of CE was observed in human TREM2 knock out iPSC-derived microglia-like cells (iMG), when these were treated with exogenous myelin (Nugent et al., 2020). The role of TREM2 in lipid metabolism is (at least in part) mediated by the enzyme phospholipase C $\gamma 2$ (PLC $\gamma 2$ ), for which a LOAD-protective variant has been identified (P522R). PLC $\gamma 2$ is an intracellular enzyme, which is also specifically expressed in microglia. The protective P522R variant is associated with a gain of function and hence loss of TREM2 or PLC $\gamma 2$ are both expected to negatively affect LOAD risk (Magno et al., 2019). Indeed, TREM2 or PLCy2 deficient iMG showed a similar defect in upregulation of genes needed for lipid metabolism. Accordingly, both TREM2- and PLCy2-deficient iPSC derived microglia fail to clear cholesterol after phagocytosis of myelin debris (Andreone et al., 2020; Nugent et al., 2020). Analysis of the lipidome after myelin treatment revealed accumulation of free cholesterol, $\mathrm{CE}$, myelin-derived ceramides [Cer, hexosylceramides (HexCer), sulfatides and diacylglycerols (DAGs) and triacylglycerols (TAGs) in the TREM2- and PLCy2-deficient iMG compared to WT iMG (Andreone et al., 2020)]. Importantly, Andreone et al. (2020) show that expression of the LOAD-protective variant P522R reduced CE accumulation to a greater extent than WT PLCy2, further indicating that TREM2 and PLCy2 work together in microglial lipid metabolism and demonstrating the relevance of this pathway for LOAD development.

Accumulation of CE was also observed in sorted microglia from $A p o E^{-/-}$mice, indicating that ApoE dependent transport prevents cholesterol overload in microglia (Nugent et al., 2020). ApoE levels are increased in $\mathrm{AD}$-brain microglia and both intracellular and extracellular clearance of $A \beta$ is greatly facilitated by lipidated ApoE particles (Jiang et al., 2008). Lipidated ApoE 
can directly interact with $A \beta$ to support phagocytosis. In addition, independent of a direct ApoE-A $\beta$ interaction, depletion of cellular cholesterol from microglia via ApoE-containing HDL particles promoted $A \beta$ degradation in primary mouse microglial cultures (Lee et al., 2012). How the TREM2-PLC $\gamma 2-A p o E$ axis contributes to LOAD development remains an important question for future studies. Whether the effects of the LOAD risk genes on $\mathrm{AD}$ pathology are directly coupled to the function of these genes in (micro)glial cholesterol metabolism is currently unknown, but of great interest to the field.

\section{From Cholesterol Dysregulation to (Micro)Gliosis}

Glial LOAD risk genes that affect (micro)glial cholesterol metabolism could impact $\mathrm{AD}$ pathology via their effect on gliosis, represented by accumulation of reactive astrocytes and immune activated microglia (Shi and Holtzman, 2018). Gliosis is detected early in AD development, and seen as a major pathological hallmark (Heneka et al., 2005; Carter et al., 2012). While considered primary a neuroprotective response, gliosis is also thought to contribute to progressive AD development (Heneka et al., 2015). Changes in cholesterol metabolism have been linked to gliosis by various studies. For example, a high cholesterol diet induces astrocytic activation and increased expression of ApoE in mice (Chen et al., 2016). In line, exogenous cholesterol addition in rat astrocytes triggered astrocyte activation, indicated by upregulation of glial fibrillary acidic protein (GFAP) (Avila-Muñoz and Arias, 2015). Finally, exogenous addition of a mixture of oxysterols, representing oxysterols that are produced when cholesterol accumulates in the $\mathrm{AD}$ brain, promote upregulation of reactive astrocyte markers, which contributed to synaptotoxicity (Staurenghi et al., 2021). In microglia, high cholesterol can affect immune function, as particularly studied in respect of cholesterol-rich myelin debris, which promotes inflammatory activation of microglia (CantutiCastelvetri et al., 2018). Myelin debris treatment in bone-marrow derived macrophages from mice caused NLRP3 inflammasome activation, possibly due to lysosomal rupture after formation of cholesterol crystals (Cantuti-Castelvetri et al., 2018). Mechanistic understanding of altered immune activation in microglia upon high cholesterol load requires further studying. In macrophages, changes in membrane cholesterol load have also been shown to affect lipid raft composition and TLR mediated signaling, where high cholesterol levels cause hyperresponsiveness to LPS treatment (Fessler and Parks, 2011). In addition, high cholesterol levels could drive CD36-dependend inflammatory signaling via inhibition of Nrf1 in the ER (Widenmaier et al., 2017). As described above reversed cholesterol transport is needed to revert the pro-inflammatory state in microglia and promote remyelination (Cantuti-Castelvetri et al., 2018). Microglia increase post-squalene sterol synthesis in response to cholesterol overload to activate LXR dependent transcription and promote cholesterol export (Berghoff et al., 2021). Increased secretion of proinflammatory cytokines IL-1 $\beta$ and IL-18 upon myelin treatment combined with TLR activation in TREM2 KO iMG suggests that increased sterol levels might indeed contribute to excessive inflammatory responses by microglia, which might be further enhanced in presence of LOAD risk genes (Andreone et al., 2020). Moreover, Lin et al. (2018) recently suggested that ApoE4 expression in microglia might be sufficient to convert them into an immune-active state. In line, ApoE4 expressing primary mouse microglia respond stronger to immune activation compared to ApoE2- or ApoE3 expressing microglia (Wong et al., 2020). Whether these processes are mediated by altered cholesterol metabolism in glia downstream of ApoE remains to be established.

\section{CONCLUSION}

Cholesterol is a central player in $\mathrm{AD}$ affecting Amyloid, Tau and gliosis. In addition, LOAD genetic risk factors point to a strong effect of lipid metabolism in $\mathrm{AD}$ development. Yet, mechanistic understanding of the pathways by which dysregulation of cholesterol metabolism contributes to $\mathrm{AD}$ development remains largely lacking. A number of major outstanding questions are; (1). How is cholesterol metabolism affected in each specific brain cell type in AD patients (2). How do LOAD-risk genes affect cholesterol metabolism in specific brain cell types and the transport of lipids between these cells (3). How do changes in brain cholesterol metabolism contribute to $\mathrm{AD}$ pathology (Amyloid, Tau, and gliosis) and finally (4) is cholesterol itself or one of its derivatives most toxic in this context?

Progress in technology development has delivered new tools to address these questions in human cells and tissue. Techniques that will help to answer the outstanding questions include the generation of human brain cell types from induced pluripotent stem cells and introduction of specific LOAD-risk mutations by CRISPR/Cas9 gene-editing (Figure 4). This approach allows the mapping of cell type specific effects of LOAD-risk variants on cholesterol metabolism combined with the possibility to mechanistically study AD pathology for a certain genetic background. Extension of this approach to co-culture or $3 \mathrm{D}$ organoid models of different iPSC derived brain cell types gives the opportunity to further study complex interplay between different cell types of the brain (Figure 4). Finally, single-nuclearor single-cell-RNA sequencing of postmortem brain tissue, or 3D iPSC derived brain cell models, with distinct LOAD-risk genotypes will result in comprehensive data on cell type specific effects on gene expression. In addition, subcellular populations that might impact cholesterol metabolism or $\mathrm{AD}$ pathology can be identified by this approach. A better mechanistic understanding of the cholesterol-dependent pathways that drive (early) AD development will also uncover novel (cell type) specific targets for rational drug discovery. An example of such a drug discovery effort for brain-lipid targeting drugs is the discovery that Efavirenz, an FDA approved HIV-drug, activates the neuronal specific enzyme CYP46A1 to promote conversion of excess cholesterol to $24 \mathrm{~S}-\mathrm{OHC}$ that can be secreted from the brain via the BBB. Efavirenz lowered pTau levels in iPSC-derived neurons from $\mathrm{AD}$ patients and improved behavior in $5 \mathrm{xFAD}$ mice 
(Petrov and Pikuleva, 2019; Petrov et al., 2019; van der Kant et al., 2019). A phase I clinical trial with intermediate-to-high doses of Efavirenz has started in patients with MCI in the United States (Nugent et al., 2020) and a similar trial with low-dose Efavirenz is planned to start in the Netherlands.

The approaches described above, and the rapidly increasing knowledge on brain lipid metabolism, will contribute to tackling the outstanding questions in this field and will undoubtedly provide much needed new insights on AD etiology and the role of cholesterol metabolism in this process. Such knowledge will likely be fundamental to develop targeted therapies to prevent, delay or cure $\mathrm{AD}$ in the future.

\section{AUTHOR CONTRIBUTIONS}

FF and RK provided the original conception and design of the manuscript and revised the manuscript. FF researched data and wrote the manuscript to which RK provided feedback.

\section{REFERENCES}

Abildayeva, K., Jansen, P. J., Hirsch-Reinshagen, V., Bloks, V. W., Bakker, A. H. F., Ramaekers, F. C. S., et al. (2006). 24(S)-hydroxycholesterol participates in a liver X receptor-controlled pathway in astrocytes that regulates apolipoprotein E-mediated cholesterol efflux. J. Biol. Chem. 281, 12799-12808. doi: 10.1074/ jbc.m601019200

Alzheimer A. (1907). Über eine eigenartige Erkrankung der Hirnrinde. Allgemeine Zeitschrift fur Psychiatrie und Psychisch-gerichtliche Medizin. 64, 146-148.

Andersson, M., Elmberger, P. O., Edlund, C., Kristensson, K., and Dallner, G. (1990). Rates of cholesterol, ubiquinone, dolichol and dolichyl-P biosynthesis in rat brain slices. FEBS Lett. 269, 15-18. doi: 10.1016/0014-5793(90)81107-y

Andreone, B. J., Przybyla, L., Llapashtica, C., Rana, A., Davis, S. S., van Lengerich, B., et al. (2020). Alzheimer's-associated PLC $\gamma 2$ is a signaling node required for both TREM2 function and the inflammatory response in human microglia. Nat. Neurosci. 23, 927-938. doi: 10.1038/s41593-020-0650-6

Anstey, K. J., Ashby-Mitchell, K., and Peters, R. (2017). Updating the Evidence on the Association between Serum Cholesterol and Risk of Late-Life Dementia: Review and Meta-Analysis. J. Alzheimers Dis. 56, 215-228. doi: 10.3233/jad160826

Arboleda-Velasquez, J. F., Lopera, F., O’Hare, M., Delgado-Tirado, S., Marino, C., Chmielewska, N., et al. (2019). Resistance to autosomal dominant Alzheimer's disease in an APOE3 Christchurch homozygote: a case report. Nat. Med. 25, 1680-1683.

Atagi, Y., Liu, C. C., Painter, M. M., Chen, X. F., Verbeeck, C., Zheng, H., et al. (2015). Apolipoprotein $\mathrm{E}$ is a ligand for triggering receptor expressed on myeloid cells 2 (TREM2). J. Biol. Chem. 290, 26043-26050. doi: 10.1074/jbc. m115.679043

Avila-Muñoz, E., and Arias, C. (2015). Cholesterol-induced astrocyte activation is associated with increased amyloid precursor protein expression and processing. Glia. 63, 2010-2022. doi: 10.1002/glia.22874

Bailey, C. C., Devaux, L. B., and Farzan, M. (2015). The triggering receptor expressed on myeloid cells 2 binds apolipoprotein E. J. Biol. Chem. 290, 26033-26042. doi: 10.1074/jbc.m115.677286

Baralla, A., Sotgiu, E., Deiana, M., Pasella, S., Pinna, S., Mannu, A., et al. (2015). Plasma Clusterin and Lipid Profile: A Link with Aging and Cardiovascular Diseases in a Population with a Consistent Number of Centenarians. PLoS One 10:e0128029. doi: 10.1371/journal.pone.0128029

Barbiellini Amidei, C., Fayosse, A., Dumurgier, J., Machado-Fragua, M. D., Tabak, A. G., van Sloten, T., et al. (2021). Association Between Age at Diabetes Onset and Subsequent Risk of Dementia. JAMA 325:1640. doi: 10.1001/jama.2021. 4001

Barrett, P. J., Song, Y., Van Horn, W. D., Hustedt, E. J., Schafer, J. M., Hadziselimovic, A., et al. (2012). The amyloid precursor protein has a flexible
Both authors contributed to the article and approved the submitted version.

\section{FUNDING}

This study was supported by grants from ZonMW-Memorabel (733050515) and Alzheimer association Nederland (WE.032019-13) to FF. RK was supported by an Alzheimer Association AARG grant (2019-AARG-643165).

\section{ACKNOWLEDGMENTS}

We would like to thank our children's grandparents (Betty, Marie-Louise, Aisha, Mahjoub, Petra, and Sjef) for the many hours of babysitting during the Covid-related lockdown while this manuscript was in preparation. Without their support this manuscript would not have come about.

transmembrane domain and binds cholesterol. Science 336, 1168-1171. doi: 10.1126/science. 1219988

Bartuzi, P., Billadeau, D. D., Favier, R., Rong, S., Dekker, D., Fedoseienko, A., et al. (2016). CCC- and WASH-mediated endosomal sorting of LDLR is required for normal clearance of circulating LDL. Nat. Commun. 7, 1-11.

Beel, A. J., Mobley, C. K., Kim, H. J., Tian, F., Hadziselimovic, A., Jap, B., et al. (2008). Structural Studies of the Transmembrane C-Terminal Domain of the Amyloid Precursor Protein (APP): Does APP Function as a Cholesterol Sensor? $\dagger+$. Biochemistry 47, 9428-9446. doi: 10.1021/bi800993c

Bellenguez, C., Küçükali, F., Jansen, I., Andrade, V., Morenau-Grau, S., Amin, N., et al. (2020). Large meta-analysis of genome-wide association studies expands knowledge of the genetic etiology of Alzheimer's disease and highlights potential translational opportunities. medRxiv 17:10.

Berghoff, S. A., Spieth, L., Sun, T., Hosang, L., Schlaphoff, L., Depp, C., et al. (2021). Microglia facilitate repair of demyelinated lesions via post-squalene sterol synthesis. Nat. Neurosci. 24, 47-60. doi: 10.1038/s41593-020-00757-6

BioRender (2021). Created with BioRender.com. Toronto,ON: BioRender.

Björkhem, I., and Meaney, S. (2004). Brain cholesterol: long secret life behind a barrier. Arterioscler. Thromb. Vasc. Biol. 24, 806-815. doi: 10.1161/01.atv. $0000120374.59826 .1 \mathrm{~b}$

Bodovitz, S., and Klein, W. L. (1996). Cholesterol Modulates $\alpha$-Secretase Cleavage of Amyloid Precursor Protein. J. Biol. Chem. 271, 4436-4440. doi: 10.1074/jbc. 271.8.4436

Brown, J., Theisler, C., Silberman, S., Magnuson, D., Gottardi-Littell, N., Lee, J. M., et al. (2004). Differential expression of cholesterol hydroxylases in Alzheimer's disease. J. Biol. Chem. 279, 34674-34681. doi: 10.1074/jbc.m40232 4200

Brown, M. S., Radhakrishnan, A., and Goldstein, J. L. (2018). Retrospective on Cholesterol Homeostasis: The Central Role of Scap [Internet]. Ann. Rev. Biochem. 87, 783-807. doi: 10.1146/annurev-biochem-062917-011852

Callaghan, M. F., Freund, P., Draganski, B., Anderson, E., Cappelletti, M., Chowdhury, R., et al. (2014). Widespread age-related differences in the human brain microstructure revealed by quantitative magnetic resonance imaging. Neurobiol. Aging 35, 1862-1872. doi: 10.1016/j.neurobiolaging.2014.02.008

Canepa, E., Borghi, R., Via, J., Traverso, N., Gambini, J., Domenicotti, C., et al. (2011). Cholesterol and amyloid- $\beta$ : Evidence for a cross-talk between astrocytes and neuronal cells. J. Alzheimer's Dis. 25, 645-653. doi: 10.3233/jad-2011110053

Cantuti-Castelvetri, L., Fitzner, D., Bosch-Queralt, M., Weil, M. T., Su, M., Sen, P., et al. (2018). Defective cholesterol clearance limits remyelination in the aged central nervous system. Science 359, 684-688. doi: 10.1126/science.aan4183

Carter, S. F., Schöll, M., Almkvist, O., Wall, A., Engler, H., Långström, B., et al. (2012). Evidence for astrocytosis in prodromal alzheimer disease provided by $11 \mathrm{C}$-deuterium-L-deprenyl: A multitracer PET paradigm combining 
11C-Pittsburgh compound B and 18F-FDG. J. Nucl. Med. 53, 37-46. doi: 10.2967/jnumed.110.087031

Castellano, J. M., Kim, J., Stewart, F. R., Jiang, H., DeMattos, R. B., Patterson, B. W., et al. (2011). Human apoE isoforms differentially regulate brain amyloid- $\beta$ peptide clearance. Sci. Transl. Med. 3:89.

Chan, R. B., Oliveira, T. G., Cortes, E. P., Honig, L. S., Duff, K. E., Small, S. A., et al. (2012). Comparative Lipidomic Analysis of Mouse and Human Brain with Alzheimer Disease. J. Biol. Chem. 287, 2678-2688. doi: 10.1074/jbc.m111. 274142

Chang, T. Y., Chang, C. C. Y., and Cheng, D. (1997). Acyl-coenzyme A: Cholesterol acyltransferase [Internet]. Vol. 66, Annual Review of Biochemistry. Annu. Rev. Biochem. 1997, 613-638. doi: 10.1146/annurev.biochem.66.1.613

Chen, J., Zhang, X., Kusumo, H., Costa, L. G., and Guizzetti, M. (2013). Cholesterol efflux is differentially regulated in neurons and astrocytes: implications for brain cholesterol homeostasis. Biochim. Biophys. Acta. 1831:263. doi: 10.1016/ j.bbalip.2012.09.007

Chen, T.-Y., Liu, P.-H., Ruan, C.-T., Chiu, L., and Kung, F.-L. (2006). The intracellular domain of amyloid precursor protein interacts with flotillin-1, a lipid raft protein. Biochem. Biophys. Res. Commun. 342, 266-272. doi: 10.1016/ j.bbrc.2006.01.156

Chen, Y. L., Wang, L. M., Chen, Y., Gao, J. Y., Marshall, C., Cai, Z. Y., et al. (2016). Changes in astrocyte functional markers and $\beta$-amyloid metabolismrelated proteins in the early stages of hypercholesterolemia. Neuroscience 316, 178-191. doi: 10.1016/j.neuroscience.2015.12.039

Chen, Y., Strickland, M. R., Soranno, A., and Holtzman, D. M. (2020). Apolipoprotein E: Structural Insights and Links to Alzheimer Disease Pathogenesis. Neuron 2020:3.

Cho, Y. Y., Kwon, O.-H., and Chung, S. (2020). Preferred Endocytosis of Amyloid Precursor Protein from Cholesterol-Enriched Lipid Raft Microdomains. Molecules 25:23. doi: 10.1042/0264-6021:3440023

Condello, C., Yuan, P., Schain, A., and Grutzendler, J. (2015). Microglia constitute a barrier that prevents neurotoxic protofibrillar $\mathrm{A} \beta 42$ hotspots around plaques. Nat. Commun. 6:6176.

Corder, E. H., Saunders, A. M., Risch, N. J., Strittmatter, W. J., Schmechel, D. E., Gaskell, P. C., et al. (1994). Protective effect of apolipoprotein E type 2 allele for late onset Alzheimer disease. Nat. Genet. 7, 180-184. doi: 10.1038/ng069 $4-180$

Corder, E. H., Saunders, A. M., Strittmatter, W. J., Schmechel, D. E., Gaskell, P. C., Small, G. W., et al. (1993). Gene dose of apolipoprotein E type 4 allele and the risk of Alzheimer's disease in late onset families. Science 261, 921-923. doi: $10.1126 /$ science. 8346443

Cordy, J. M., Hussain, I., Dingwall, C., Hooper, N. M., and Turner, A. J. (2003). Exclusively targeting $\beta$-secretase to lipid rafts by GPI-anchor addition upregulates $\beta$-site processing of the amyloid precursor protein. Proc. Natl. Acad. Sci. U S A. 100, 11735-11740. doi: 10.1073/pnas.1635130100

Cossec, J. C., Simon, A., Marquer, C., Moldrich, R. X., Leterrier, C., Rossier, J., et al. (2010). Clathrin-dependent APP endocytosis and A $\beta$ secretion are highly sensitive to the level of plasma membrane cholesterol. Biochim. Biophys. ActaMol. Cell Biol. Lipids. 1801, 846-852. doi: 10.1016/j.bbalip.2010.05.010

Cramer, C., Haan, M. N., Galea, S., Langa, K. M., and Kalbfleisch, J. D. (2008). Use of statins and incidence of dementia and cognitive impairment without dementia in a cohort study. Neurology 71, 344-350. doi: 10.1212/01.wnl. 0000319647.15752.7b

Crisby, M., Rahman, S. M. A., Sylvén, C., Winblad, B., and Schultzberg, M. (2004). Effects of high cholesterol diet on gliosis in apolipoprotein E knockout mice. Implications for Alzheimer's disease and stroke. Neurosci. Lett. 369, 87-92. doi: 10.1016/j.neulet.2004.05.057

Cruchaga, C., Kauwe, J. S. K., Nowotny, P., Bales, K., Pickering, E. H., Mayo, K., et al. (2012). Cerebrospinal fluid APOE levels: An endophenotype for genetic studies for Alzheimer's disease. Hum. Mol. Genet. 21, 4558-4571. doi: 10.1093/ $\mathrm{hmg} / \mathrm{dds} 296$

Cutler, R. G., Kelly, J., Storie, K., Pedersen, W. A., Tammara, A., Hatanpaa, K., et al. (2004). Involvement of oxidative stress-induced abnormalities in ceramide and cholesterol metabolism in brain aging and Alzheimer's disease. Proc. Natl. Acad. Sci. U S A. 101, 2070. doi: 10.1073/pnas.0305799101

Dai, L., Zou, L., Meng, L., Qiang, G., Yan, M., and Zhang, Z. (2021). Cholesterol Metabolism in Neurodegenerative Diseases: Molecular Mechanisms and Therapeutic Targets. Mol. Neurobiol. 7, 1-19.
Decock, M., El Haylani, L., Stanga, S., Dewachter, I., Octave, J. N., Smith, S. O., et al. (2015). Analysis by a highly sensitive split luciferase assay of the regions involved in APP dimerization and its impact on processing. FEBS Open Bio. 5, 763-773. doi: 10.1016/j.fob.2015.09.002

Del-Aguila, J. L., Li, Z., Dube, U., Mihindukulasuriya, K. A., Budde, J. P., Fernandez, M. V., et al. (2019). A single-nuclei RNA sequencing study of Mendelian and sporadic AD in the human brain. Alzheimers Res. Ther. 11:71.

Dietschy, J. M., and Turley, S. D. (2004). Thematic review series: Brain Lipids. Cholesterol metabolism in the central nervous system during early development and in the mature animal. J. Lipid. Res. 45, 1375-1397. doi: 10.1194/jlr.r400004-jlr200

Efthymiou, A. G., and Goate, A. M. (2017). Late onset Alzheimer's disease genetics implicates microglial pathways in disease risk [Internet]. Mole. Neurodegener. 2017:12.

Eggert, S., Gonzalez, A. C., Thomas, C., Schilling, S., Schwarz, S. M., Tischer, C., et al. (2018). Dimerization leads to changes in APP (amyloid precursor protein) trafficking mediated by LRP1 and SorLA. Cell Mol. Life Sci. 75, 301-322. doi: 10.1007/s00018-017-2625-7

Eggert, S., Midthune, B., Cottrell, B., and Koo, E. H. (2009). Induced dimerization of the amyloid precursor protein leads to decreased amyloid- $\beta$ protein production. J. Biol. Chem. 284, 28943-28952. doi: 10.1074/jbc.m109.038646

Ehehalt, R., Keller, P., Haass, C., Thiele, C., and Simons, K. (2003). Amyloidogenic processing of the Alzheimer $\beta$-amyloid precursor protein depends on lipid rafts. J. Cell Biol. 160, 113-123. doi: 10.1083/jcb.200207113

Fabelo, N., Martín, V., Marín, R., Moreno, D., Ferrer, I., and Díaz, M. (2014). Altered lipid composition in cortical lipid rafts occurs at early stages of sporadic Alzheimer's disease and facilitates APP/BACE1 interactions. Neurobiol. Aging. 35, 1801-1812. doi: 10.1016/j.neurobiolaging.2014.02.005

Farmer, B. C., Kluemper, J., and Johnson, L. A. (2019). Apolipoprotein E4 Alters Astrocyte Fatty Acid Metabolism and Lipid Droplet Formation. Cells 8:2.

Farmer, B. C., Walsh, A. E., Kluemper, J. C., and Johnson, L. A. (2020). Lipid Droplets in Neurodegenerative Disorders. Front. Neurosci. 14:742.

Fedoseienko, A., Wijers, M., Wolters, J. C., Dekker, D., Smit, M., Huijkman, N., et al. (2018). The COMMD family regulates plasma LDL levels and attenuates atherosclerosis through stabilizing the CCC complex in endosomal LDLR traffcking. Circ. Res. 122, 1648-1660. doi: 10.1161/circresaha.117.312004

Fessler, M. B., and Parks, J. S. (2011). Intracellular lipid flux and membrane microdomains as organizing principles in inflammatory cell signaling. J. Immunol. 187, 1529-1535. doi: 10.4049/jimmunol.1100253

Fong, L. K., Yang, M. M., Chaves, R., dos, S., Reyna, S. M., Langness, V. F., et al. (2018). Full-length amyloid precursor protein regulates lipoprotein metabolism and amyloid- clearance in human astrocytes. J. Biol. Chem. 293, 11341-11357. doi: 10.1074/jbc.ra117.000441

Foster, E. M., Dangla-Valls, A., Lovestone, S., Ribe, E. M., and Buckley, N. J. (2019). Clusterin in Alzheimer's Disease: Mechanisms, Genetics, and Lessons From Other Pathologies. Front. Neurosci. 13:164. doi: 10.1097/00002093-19880203000012

Fryer, J. D., DeMattos, R. B., McCormick, L. M., O’Dell, M. A., Spinner, M. L., Bales, K. R., et al. (2005). The low density lipoprotein receptor regulates the level of central nervous system human and murine apolipoprotein $\mathrm{E}$ but does not modify amyloid plaque pathology in PDAPP mice. J. Biol. Chem. 280, 25754-25759. doi: 10.1074/jbc.m502143200

Fünfschilling, U., Saher, G., Xiao, L., Möbius, W., and Nave, K.-A. (2007). Survival of adult neurons lacking cholesterol synthesis in vivo. BMC Neurosci. 8:1.

Gamba, P., Testa, G., Gargiulo, S., Staurenghi, E., Poli, G., and Leonarduzzi, G. (2015). Oxidized cholesterol as the driving force behind the development of Alzheimer's disease. Front. Aging Neurosci. 7:119.

Gelissen, I. C., Harris, M., Rye, K. A., Quinn, C., Brown, A. J., Kockx, M., et al. (2006). ABCA1 and ABCG1 synergize to mediate cholesterol export to ApoA-I. Arterioscler. Thromb. Vasc. Biol. 26, 534-540. doi: 10.1161/01.atv.0000200082. 58536.e1

Gelissen, I. C., Hochgrebe, T., Wilson, M. R., Easterbrook-Smith, S. B., Jessup, W., Dean, R. T., et al. (1998). Apolipoprotein J (clusterin) induces cholesterol export from macrophage-foam cells: a potential anti-atherogenic function? Biochem. J. 331, 231-237. doi: 10.1042/bj3310231

Genaro-Mattos, T. C., Anderson, A., Allen, L. B., Korade, Z., and Mirnics, K. (2019). Cholesterol Biosynthesis and Uptake in Developing Neurons. ACS Chem. Neurosci. 10, 3671-3681. doi: 10.1021/acschemneuro.9b00248 
Gilardi, F., Viviani, B., Galmozzi, A., Boraso, M., Bartesaghi, S., Torri, A., et al. (2009). Expression of sterol 27-hydroxylase in glial cells and its regulation by liver X receptor signaling. Neuroscience 164, 530-540. doi: 10.1016/j. neuroscience.2009.08.003

Glöckner, F., Meske, V., Lütjohann, D., and Ohm, T. G. (2011). Dietary cholesterol and its effect on tau protein: A study in apolipoprotein e-deficient and P301L human tau mice. J. Neuropathol. Exp. Neurol. 70, 292-301. doi: 10.1097/nen. 0b013e318212f185

Goldstein, J. L., and Brown, M. S. (2009). The LDL receptor [Internet]. Vol. 29, Arteriosclerosis, Thrombosis, and Vascular Biology. Arterioscler. Thromb. Vasc. Biol. 2009, 431-438. doi: 10.1161/atvbaha.108.179564

Grimm, M. O. W., Grimm, H. S., Tomic, I., Beyreuther, K., Hartmann, T., and Bergmann, C. (2008). Independent Inhibition of Alzheimer Disease $\beta$ and $\gamma$-Secretase Cleavage by Lowered Cholesterol Levels. J. Biol. Chem. 283, 11302-11311. doi: 10.1074/jbc.m801520200

Griswold, A. J., Celis, K., Bussies, P. L., Rajabli, F., and Whitehead, P. L. (2021). Increased APOE $\varepsilon 4$ expression is associated with the difference in Alzheimer's disease risk from diverse ancestral backgrounds. Alzheimer's Dement 2021:12287.

Grubman, A., Chew, G., Ouyang, J. F., Sun, G., Choo, X. Y., McLean, C., et al. (2019). A single-cell atlas of entorhinal cortex from individuals with Alzheimer's disease reveals cell-type-specific gene expression regulation. Nat. Neurosci. 22, 2087-2097. doi: 10.1038/s41593-019-0539-4

Haag, M. D. M., Hofman, A., Koudstaal, P. J., Stricker, B. H. C., and Breteler, M. M. B. (2009). Statins are associated with a reduced risk of Alzheimer disease regardless of lipophilicity. The Rotterdam Study. J. Neurol. Neurosurg. Psychiatry. 80, 13-17. doi: 10.1136/jnnp.2008.150433

Hammond, T. R., Dufort, C., Dissing-Olesen, L., Giera, S., Young, A., Wysoker, A., et al. (2019). Single cell RNA sequencing of microglia throughout the mouse lifespan and in the injured brain reveals complex cell-state changes. Immunity 50:253. doi: 10.1016/j.immuni.2018.11.004

Heinsinger, N. M., Gachechiladze, M. A., and Rebeck, G. W. (2016). Apolipoprotein e genotype affects size of ApoE complexes in cerebrospinal fluid. J. Neuropathol. Exp. Neurol. 75, 918-924. doi: 10.1093/jnen/nlw067

Heneka, M. T., Carson, M. J., Khoury, J. E., Landreth, G. E., Brosseron, F., Feinstein, D. L., et al. (2015). Neuroinflammation in Alzheimer's disease. Lancet Neurol. 14, 388-405.

Heneka, M. T., Sastre, M., Dumitrescu-Ozimek, L., Dewachter, I., Walter, J., Klockgether, T., et al. (2005). Focal glial activation coincides with increased BACE1 activation and precedes amyloid plaque deposition in APP[V717I] transgenic mice. J. Neuroinflammation 2:22.

Heverin, M., Bogdanovic, N., Lütjohann, D., Bayer, T., Pikuleva, I., Bretillon, L., et al. (2004). Changes in the levels of cerebral and extracerebral sterols in the brain of patients with Alzheimer's disease. J. Lipid. Res. 45, 186-193. doi: 10.1194/jlr.m300320-jlr200

Hicks, D. A., Nalivaeva, N. N., and Turner, A. J. (2012). Lipid rafts and Alzheimer's disease: Protein-lipid interactions and perturbation of signaling. Front. Physiol. 2012:3.

Hu, J., Liu, C. C., Chen, X. F., Zhang, Y. W., Xu, H., and Bu, G. (2015). Opposing effects of viral mediated brain expression of apolipoprotein E2 (apoE2) and apoE4 on apoE lipidation and $\mathrm{A} \beta$ metabolism in apoE4-targeted replacement mice. Mol. Neurodegener. 10:1. doi: 10.1016/j.bbr.2004.09.019

Huang, Y. (2010). Mechanisms linking apolipoprotein E isoforms with cardiovascular and neurological diseases. Curr. Opin. Lipidol. 21, 337-345. doi: 10.1097/mol.0b013e32833af368

Huang, Y., Weisgraber, K. H., Mucke, L., and Mahley, R. W. (2004). Apolipoprotein E: diversity of cellular origins, structural and biophysical properties, and effects in Alzheimer's disease. J. Mol. Neurosci. 23, 189-204. doi: 10.1385/jmn:23:3:189

Hutter-Paier, B., Huttunen, H. J., Puglielli, L., Eckman, C. B., Kim, D. Y., Hofmeister, A., et al. (2004). The ACAT inhibitor CP-113,818 markedly reduces amyloid pathology in a mouse model of Alzheimer's disease. Neuron. 44, 227-238. doi: 10.1016/j.neuron.2004.08.043

Huttunen, H. J., Peach, C., Bhattacharyya, R., Barren, C., Pettingell, W., HutterPaier, B., et al. (2009). Inhibition of acyl-coenzyme A: cholesterol acyl transferase modulates amyloid precursor protein trafficking in the early secretory pathway. FASEB J. 23, 3819-3828. doi: 10.1096/fj.09-134999

Igbavboa, U., Sun, G. Y., Weisman, G. A., He, Y., and Wood, W. G. (2009). Amyloid beta-protein stimulates trafficking of cholesterol and caveolin-1 from the plasma membrane to the Golgi complex in mouse primary astrocytes. Neuroscience 162, 328-338. doi: 10.1016/j.neuroscience.2009.04.049

Ikonen, E. (2008). Cellular cholesterol trafficking and compartmentalization. Nat. Rev. Mol. Cell Biol. 9, 125-138. doi: 10.1038/nrm2336

Ioannou, M. S., Jackson, J., Sheu, S.-H., Chang, C.-L., Weigel, A. V., Liu, H., et al. (2019). Neuron-Astrocyte Metabolic Coupling Protects against ActivityInduced Fatty Acid Toxicity. Cell 177, 1522.e-1535.e.

Iwamoto, N., Abe-Dohmae, S., Sato, R., and Yokoyama, S. (2006). ABCA7 expression is regulated by cellular cholesterol through the SREBP2 pathway and associated with phagocytosis. J. Lipid Res. 47, 1915-1927. doi: 10.1194/ jlr.m600127-jlr200

Jansen, I. E., Savage, J. E., Watanabe, K., Bryois, J., Williams, D. M., Steinberg, S., et al. (2019). Genome-wide meta-analysis identifies new loci and functional pathways influencing Alzheimer's disease risk. Nat. Genet. 51, 404-413.

Jeong, W., Lee, H., Cho, S., and Seo, J. (2019). ApoE4-Induced Cholesterol Dysregulation and Its Brain Cell Type-Specific Implications in the Pathogenesis of Alzheimer's Disease [Internet]. Mole. Cells 42, 739-746.

Jiang, Q., Lee, C. Y. D., Mandrekar, S., Wilkinson, B., Cramer, P., Zelcer, N., et al. (2008). ApoE Promotes the Proteolytic Degradation of A $\beta$. Neuron. 58, 681-693. doi: 10.1016/j.neuron.2008.04.010

Jick, H., Zornberg, G. L., Jick, S. S., Seshadri, S., and Drachman, D. A. (2000). Statins and the risk of dementia. Lancet 356, 1627-1631.

Jin, P., Pan, Y., Pan, Z., Xu, J., Lin, M., Sun, Z., et al. (2018). Alzheimer-like brain metabolic and structural features in cholesterol-fed rabbit detected by magnetic resonance imaging. Lipids Health Dis. 17:61.

Jongbloed, W., van Dijk, K. D., Mulder, S. D., van de Berg, W. D. J., Blankenstein, M. A., van der Flier, W., et al. (2015). Clusterin Levels in Plasma Predict Cognitive Decline and Progression to Alzheimer's Disease. J. Alzheimers Dis. 46, 1103-1110. doi: 10.3233/jad-150036

Julia, T. C. W., Liang, S. A., Qian, L., Pipalia, N. H., Chao, M. J., Shi, Y., et al. (2019). Cholesterol and matrisome pathways dysregulated in human APOE $\varepsilon 4$ glia [Internet]. bioRxiv 2019:713362.

Kaden, D., Munter, L. M., Joshi, M., Treiber, C., Weise, C., Bethge, T., et al. (2008). Homophilic interactions of the amyloid precursor protein (APP) ectodomain are regulated by the loop region and affect $\beta$-secretase cleavage of APP. J. Biol. Chem. 283, 7271-7279. doi: 10.1074/jbc.m708046200

Kaminski, W. E., Orsó, E., Diederich, W., Klucken, J., Drobnik, W., and Schmitz, G. (2000). Identification of a novel human sterol-sensitive ATP-binding cassette transporter (ABCA7). Biochem. Biophys. Res. Commun. 273, 532-538. doi: 10.1006/bbrc.2000.2954

Kant, R., Goldstein, L. S. B., and Ossenkoppele, R. (2019). Amyloid- $\beta$-independent regulators of tau pathology in Alzheimer disease. Nat. Rev. Neurosci. 8, 1-15. doi: $10.2174 / 1567211212443562050$

Keren-Shaul, H., Spinrad, A., Weiner, A., Matcovitch-Natan, O., Dvir-Szternfeld, R., Ulland, T. K., et al. (2017). A Unique Microglia Type Associated with Restricting Development of Alzheimer's Disease. Cell 169, 1276.e-1290.e.

Kojro, E., Gimpl, G., Lammich, S., Marz, W., and Fahrenholz, F. (2001). Low cholesterol stimulates the nonamyloidogenic pathway by its effect on the alpha -secretase ADAM 10. Proc. Natl. Acad. Sci. U S A. 98, 5815-5820. doi: 10.1073/ pnas.081612998

Koldamova, R. P., Lefterov, I. M., Ikonomovic, M. D., Skoko, J., Lefterov, P. I., Isanski, B. A., et al. (2003). 22R-Hydroxycholesterol and 9-cis-Retinoic Acid Induce ATP-binding Cassette Transporter A1 Expression and Cholesterol Efflux in Brain Cells and Decrease Amyloid $\beta$ Secretion. J. Biol. Chem. 278, 13244-13256. doi: 10.1074/jbc.m300044200

Krimbou, L., Denis, M., Haidar, B., Carrier, M., Marcil, M., and Genest, J. (2004). Molecular interactions between apoE and ABCA1: Impact on apoE lipidation. J. Lipid. Res. 45, 839-848. doi: 10.1194/jlr.m300418-jlr200

Kunkle, B. W., Grenier-Boley, B., Sims, R., Bis, J. C., Damotte, V., Naj, A. C., et al. (2019). Genetic meta-analysis of diagnosed Alzheimer's disease identifies new risk loci and implicates $A \beta$, tau, immunity and lipid processing. Nat. Genet. 51, 414-430.

Kwon, H. J., Abi-Mosleh, L., Wang, M. L., Deisenhofer, J., Goldstein, J. L., Brown, M. S., et al. (2009). Structure of N-Terminal Domain of NPC1 Reveals Distinct Subdomains for Binding and Transfer of Cholesterol. Cell 137, 1213-1224. doi: 10.1016/j.cell.2009.03.049

Lambert, J. C., Ibrahim-Verbaas, C. A., Harold, D., Naj, A. C., Sims, R., Bellenguez, C., et al. (2013). Meta-analysis of 74,046 individuals 
identifies 11 new susceptibility loci for Alzheimer's disease. Nat. Genet. 45, $1452-1458$.

Langness, V. F., van der Kant, R., Das, U., Wang, L., and Chaves, R. (2021). Cholesterol-lowering drugs reduce APP processing to A $\beta$ by inducing APP dimerization. Mol. Biol. Cell 32, 247-259. doi: 10.1091/mbc.e20-05-0345

Le, L. T. M., Thompson, J. R., Aikawa, T., Kanikeyo, T., and Alam, A. (2021). CryoEM structure of lipid embedded human ABCA7 at 3.6 resolution. bioRxiv 2021:433448.

Ledreux, A., Wang, X., Schultzberg, M., Granholm, A. C., and Freeman, L. R. (2016). Detrimental effects of a high fat/high cholesterol diet on memory and hippocampal markers in aged rats. Behav. Brain Res. 312, 294-304. doi: 10. 1016/j.bbr.2016.06.012

Lee, C. Y. D., Tse, W., Smith, J. D., and Landreth, G. E. (2012). Apolipoprotein E Promotes-Amyloid Trafficking and Degradation by Modulating Microglial Cholesterol Levels. J. Biol. Chem. 287, 2032-2044. doi: 10.1074/jbc.m111. 295451

Leyns, C. E. G., Gratuze, M., Narasimhan, S., Jain, N., Koscal, L. J., Jiang, H., et al. (2019). TREM2 function impedes tau seeding in neuritic plaques. Nat. Neurosci. 22, 1217-1222. doi: 10.1038/s41593-019-0433-0

Li, G., Shofer, J. B., Rhew, I. C., Kukull, W. A., Peskind, E. R., McCormick, W., et al. (2010). Age-varying association between statin use and incident Alzheimer's disease. J. Am. Geriatr. Soc. 58, 1311-1317. doi: 10.1111/j.1532-5415.2010. 02906. $\mathrm{x}$

Li, L., Zeng, F., Liu, Y. H., Li, H. Y., Dong, S. Y., Peng, Z. Y., et al. (2018). CYP46A1 and the APOE 4 Allele Polymorphisms Correlate with the Risk of Alzheimer's Disease. Mol. Neurobiol. 55, 8179-8187. doi: 10.1007/s12035-018-0952-9

Li, X., Zhang, J., Li, D., He, C., He, K., Xue, T., et al. (2021). Astrocytic ApoE reprograms neuronal cholesterol metabolism and histone-acetylation-mediated memory. Neuron 2021:26.

Lin, F.-C., Chuang, Y.-S., Hsieh, H.-M., Lee, T.-C., Chiu, K.-F., Liu, C.-K., et al. (2015). Early Statin Use and the Progression of Alzheimer Disease: A Total Population-Based Case-Control Study. Medicine 94, e2143. doi: 10.1097/md. 0000000000002143

Lin, Y.-T., Seo, J., Gao, F., Feldman, H. M., Wen, H.-L., Penney, J., et al. (2018). APOE4 Causes Widespread Molecular and Cellular Alterations Associated with Alzheimer's Disease Phenotypes in Human iPSC-Derived Brain Cell Types. Neuron 98, 1141.e-1154.e.

Liu, C. C., Liu, C. C., Kanekiyo, T., Xu, H., and Bu, G. (2013). Apolipoprotein E and Alzheimer disease: risk, mechanisms and therapy. Nat. Rev. Neurol. 9:2.

Liu, L., MacKenzie, K. R., Putluri, N., Maletić-Savatić, M., and Bellen, H. J. (2017). The Glia-Neuron Lactate Shuttle and Elevated ROS Promote Lipid Synthesis in Neurons and Lipid Droplet Accumulation in Glia via APOE/D. Cell Metab. 26, 719.e-737.e.

Liu, L., Zhang, K., Sandoval, H., Yamamoto, S., Jaiswal, M., Sanz, E., et al. (2015). Glial Lipid Droplets and ROS Induced by Mitochondrial Defects Promote Neurodegeneration. Cell 160, 177-190. doi: 10.1016/j.cell.2014.12.019

Liu, Q., Zerbinatti, C. V., Zhang, J., Hoe, H. S., Wang, B., Cole, S. L., et al. (2007). Amyloid Precursor Protein Regulates Brain Apolipoprotein E and Cholesterol Metabolism through Lipoprotein Receptor LRP1. Neuron 56, 66-78. doi: 10. 1016/j.neuron.2007.08.008

Loving, B. A., and Bruce, K. D. (2020). Lipid and Lipoprotein Metabolism in Microglia. Front. Physiol. 11:393.

Lund, E. G., Guileyardo, J. M., and Russell, D. W. (1999). cDNA cloning of cholesterol 24-hydroxylase, a mediator of cholesterol homeostasis in the brain. Proc. Natl. Acad. Sci. 96, 7238-7243. doi: 10.1073/pnas.96.13.7238

Lund, E. G., Xie, C., Kotti, T., Turley, S. D., Dietschy, J. M., and Russell, D. W. (2003). Knockout of the Cholesterol 24-Hydroxylase Gene in Mice Reveals a Brain-specific Mechanism of Cholesterol Turnover. J. Biol. Chem. 278, 22980 22988. doi: $10.1074 / \mathrm{jbc} . \mathrm{m} 303415200$

Luo, J., Yang, H., and Song, B.-L. (2019). Mechanisms and regulation of cholesterol homeostasis. Nat. Rev. Mol. Cell Biol. 2019:17.

Lütjohann, D., Breuer, O., Ahlborg, G., Nennesmo, I., Sidén, A., Diczfalusy, U., et al. (1996). Cholesterol homeostasis in human brain: evidence for an agedependent flux of 24S-hydroxycholesterol from the brain into the circulation. Proc. Natl. Acad. Sci. 93, 9799-9804. doi: 10.1073/pnas.93.18.9799

Lütjohann, D., Papassotiropoulos, A., Björkhem, I., Locatelli, S., Bagli, M., Oehring, R. D., et al. (2000). Plasma 24S-hydroxycholesterol (cerebrosterol) is increased in Alzheimer and vascular demented patients. J. Lipid Res. 41, 195-198. doi: $10.1016 / \mathrm{s} 0022-2275(20) 32052-6$
Lyssenko, N. N., and Praticò, D. (2020). ABCA7 and the altered lipidostasis hypothesis of Alzheimer's disease. Alzheimer's Dement 17:12220.

Ma, Y., Ajnakina, O., Steptoe, A., and Cadar, D. (2020). Higher risk of dementia in English older individuals who are overweight or obese. Int. J. Epidemiol. 49, 1353-1365. doi: 10.1093/ije/dyaa099

Magno, L., Lessard, C. B., Martins, M., Lang, V., Cruz, P., Asi, Y., et al. (2019). Alzheimer's disease phospholipase C-gamma-2 (PLCG2) protective variant is a functional hypermorph. Alzheimers Res. Ther. 11:16.

Mahley, R. W. (2016). Apolipoprotein E: from cardiovascular disease to neurodegenerative disorders. J. Mol. Med. 94, 739-746. doi: 10.1007/s00109016-1427-y

Marquer, C., Devauges, V., Cossec, J., Liot, G., Lécart, S., Saudou, F., et al. (2011). Local cholesterol increase triggers amyloid precursor protein-Bacel clustering in lipid rafts and rapid endocytosis. FASEB J. 25, 1295-1305. doi: 10.1096/fj.10168633

Marquer, C., Laine, J., Dauphinot, L., Hanbouch, L., Lemercier-Neuillet, C., Pierrot, N., et al. (2014). Increasing membrane cholesterol of neurons in culture recapitulates Alzheimer's disease early phenotypes. Mol. Neurodegener. 9:60. doi: 10.1186/1750-1326-9-60

Marschallinger, J., Iram, T., Zardeneta, M., Lee, S. E., Lehallier, B., Haney, M. S., et al. (2020). Lipid-droplet-accumulating microglia represent a dysfunctional and proinflammatory state in the aging brain. Nat. Neurosci. 23, 194-208. doi: 10.1038/s41593-019-0566-1

Mathys, H., Davila-Velderrain, J., Peng, Z., Gao, F., Mohammadi, S., Young, J. Z., et al. (2019). Single-cell transcriptomic analysis of Alzheimer's disease HHS Public Access. Nature 570, 332-337.

Matsuda, A., Nagao, K., Matsuo, M., Kioka, N., and Ueda, K. (2013). 24(S)hydroxycholesterol is actively eliminated from neuronal cells by ABCA1. J. Neurochem. 126, 93-101. doi: 10.1111/jnc. 12275

Mauch, D. H., Nägler, K., Schumacher, S., Göritz, C., Müller, E.-C., Otto, A., et al. (2001). CNS Synaptogenesis Promoted by Glia-Derived Cholesterol. Science 294, 1354-1357. doi: 10.1126/science.294.5545.1354

McQuade, A., and Blurton-Jones, M. (2019). Microglia in Alzheimer's Disease: Exploring How Genetics and Phenotype Influence Risk. J. Mol. Biol. 2019:7.

Michikawa, M., Fan, Q.-W., Isobe, I., and Yanagisawa, K. (2000). Apolipoprotein E Exhibits Isoform-Specific Promotion of Lipid Efflux from Astrocytes and Neurons in Culture. J. Neurochem. 74, 1008-1016. doi: 10.1046/j.1471-4159. 2000.0741008.x

Montagne, A., Barnes, S. R., Sweeney, M. D., Halliday, M. R., Sagare, A. P., Zhao, Z., et al. (2015). Blood-Brain Barrier Breakdown in the Aging Human Hippocampus. Neuron. 85, 296-302. doi: 10.1016/j.neuron.2014.12.032

Montesinos, J., Pera, M., Larrea, D., Guardia-Laguarta, C., Agrawal, R. R., Velasco, K. R., et al. (2020). The Alzheimer's disease-associated C99 fragment of APP regulates cellular cholesterol trafficking. EMBO J. 15:39.

Mori, T., Paris, D., Town, T., Rojiani, A. M., Sparks, D. L., Delledonne, A., et al. (2001). Cholesterol accumulates in senile plaques of Alzheimer disease patients and in transgenic APP(SW) mice. J. Neuropathol. Exp. Neurol. 60, 778-785. doi: 10.1093/jnen/60.8.778

Moulton, M. J., Barish, S., Ralhan, I., Chang, J., Goodman, L. D., Harland, J. G., et al. (2021). Neuronal ROS-Induced Glial Lipid Droplet Formation is Altered by Loss of Alzheimer's Disease-associated Genes. bioRxiv 2021:433580.

Nierzwicki, $€$, and Czub, J. (2015). Specific Binding of Cholesterol to the Amyloid Precursor Protein: Structure of the Complex and Driving Forces Characterized in Molecular Detail. J. Phys. Chem. Lett. 6, 784-790. doi: 10.1021/acs.jpclett. 5 b00197

Nieweg, K., Schaller, H., and Pfrieger, F. W. (2009). Marked differences in cholesterol synthesis between neurons and glial cells from postnatal rats. J. Neurochem. 109:2009.

Nugent, A. A., Lin, K., van Lengerich, B., Lianoglou, S., Przybyla, L., Davis, S. S., et al. (2020). TREM2 Regulates Microglial Cholesterol Metabolism upon Chronic Phagocytic Challenge. Neuron 105, 837.e-854.e.

Olah, M., Menon, V., Habib, N., Taga, M. F., Ma, Y., Yung, C. J., et al. (2020). Single cell RNA sequencing of human microglia uncovers a subset associated with Alzheimer's disease. Nat. Commun. 11:1.

Olsen, B. N., Schlesinger, P. H., Ory, D. S., and Baker, N. A. (2012). Side-chain oxysterols: from cells to membranes to molecules. Biochim. Biophys. Acta. 1818, 330-336. doi: 10.1016/j.bbamem.2011.06.014

Osenkowski, P., Ye, W., Wang, R., Wolfe, M. S., and Selkoe, D. J. (2008). Direct and potent regulation of $\gamma$-secretase by its lipid 
microenvironment. J. Biol. Chem. 283, 22529-22540. doi: 10.1074/jbc.m80192 5200

Papassotiropoulos, A., Lütjohann, D., Bagli, M., Locatelli, S., Jessen, F., Buschfort, R., et al. (2002). 24S-hydroxycholesterol in cerebrospinal fluid is elevated in early stages of dementia. J. Psychiatr. Res. 36, 27-32. doi: 10.1016/s00223956(01)00050-4

Pappolla, M. A., Bryant-Thomas, T. K., Herbert, D., Pacheco, J., Garcia, M. F., Manjon, M., et al. (2003). Mild hypercholesterolemia is an early risk factor for the development of Alzheimer amyloid pathology. Neurology 61, 199-205. doi: 10.1212/01.wnl.0000070182.02537.84

Pedditizi, E., Peters, R., and Beckett, N. (2016). The risk of overweight/obesity in mid-life and late life for the development of dementia: a systematic review and meta-analysis of longitudinal studies. Age Ageing 45, 14-21. doi: 10.1093/ ageing/afv151

Petrov, A. M., and Pikuleva, I. A. (2019). Cholesterol 24-Hydroxylation by CYP46A1: Benefits of Modulation for Brain Diseases. Neurotherapeutics 16, 635-648. doi: 10.1007/s13311-019-00731-6

Petrov, A. M., Lam, M., Mast, N., Moon, J., Li, Y., Maxfield, E., et al. (2019). CYP46A1 Activation by Efavirenz Leads to Behavioral Improvement without Significant Changes in Amyloid Plaque Load in the Brain of 5XFAD Mice. Neurotherapeutics 16, 710-724. doi: 10.1007/s13311-019-00737-0

Pfrieger, F. W. (2003). Role of cholesterol in synapse formation and function. Biochim. Biophys. Acta - Biomembr. 1610, 271-280. doi: 10.1016/s00052736(03)00024-5

Pfrieger, F. W., and Ungerer, N. (2011). Cholesterol metabolism in neurons and astrocytes. Prog. Lipid Res. 50, 357-371. doi: 10.1016/j.plipres.2011.06.002

Pierrot, N., Tyteca, D., D'auria, L., Dewachter, I., Gailly, P., Hendrickx, A., et al. (2013). Amyloid precursor protein controls cholesterol turnover needed for neuronal activity. EMBO Mol. Med. 5, 608-625. doi: 10.1002/emmm. 201202215

Pimenova, A. A., Raj, T., and Goate, A. M. (2018). Untangling Genetic Risk for Alzheimer's Disease. Biol. Psychiatry. 83, 300-310. doi: 10.1016/j.biopsych. 2017.05.014

Prince, M., Wimo, A. G. M., Ali, G. C., Wu, Y. T., and Prina, M. (2015). World Alzheimer Report 2015: the global impact of dementia: an analysis of prevalence, incidence, cost and trends. London: Alzheimer's Disease International.

Puglielli, L., Konopka, G., Pack-Chung, E., Ingano, L. A. M., Berezovska, O., Hyman, B. T., et al. (2001). Acyl-coenzyme A: cholesterol acyltransferase modulates the generation of the amyloid $\beta$-peptide. Nat. Cell Biol. 3, 905-912. doi: 10.1038/ncb1001-905

Qi, G., Mi, Y., Shi, X., Gu, H., Brinton, R. D., and Yin, F. (2021). ApoE4 Impairs Neuron-Astrocyte Coupling of Fatty Acid Metabolism. Cell Rep. 34, 108572. doi: 10.1016/j.celrep.2020.108572

Quan, G., Xie, C., Dietschy, J. M., and Turley, S. D. (2003). Ontogenesis and regulation of cholesterol metabolism in the central nervous system of the mouse. Brain Res. Dev. Brain Res. 146, 87-98. doi: 10.1016/j.devbrainres.2003. 09.015

Radhakrishnan, A., Ikeda, Y., Kwon, H. J., Brown, M. S., and Goldstein, J. L. (2007). Sterol-regulated transport of SREBPs from endoplasmic reticulum to Golgi: oxysterols block transport by binding to Insig. Proc. Natl. Acad. Sci. U S A. 104, 6511-6518. doi: 10.1073/pnas.0700899104

Rahman, A., Akterin, S., Flores-Morales, A., Crisby, M., Kivipelto, M., Schultzberg, M., et al. (2005). High cholesterol diet induces tau hyperphosphorylation in apolipoprotein e deficient mice. FEBS Lett. 579, 6411-6416. doi: 10.1016/j. febslet.2005.10.024

Ramirez, D. M. O., Andersson, S., and Russell, D. W. (2008). Neuronal expression and subcellular localization of cholesterol 24-hydroxylase in the mouse brain. J. Comp. Neurol. 507, 1676-1693. doi: 10.1002/cne.21605

Rawat, V., Wang, S., Sima, J., Bar, R., Liraz, O., Gundimeda, U., et al. (2019). ApoE4 Alters ABCA1 Membrane Trafficking in Astrocytes. J. Neurosci. 39, 9611-9622. doi: 10.1523/jneurosci.1400-19.2019

Refolo, L. M., Malester, B., LaFrancois, J., Bryant-Thomas, T., Wang, R., Tint, G. S., et al. (2000). Hypercholesterolemia accelerates the Alzheimer's amyloid pathology in a transgenic mouse model. Neurobiol. Dis. 7:4.

Refolo, L. M., Pappolla, M. A., LaFrancois, J., Malester, B., Schmidt, S. D., ThomasBryant, T., et al. (2001). A Cholesterol-Lowering Drug Reduces $\beta$-Amyloid Pathology in a Transgenic Mouse Model of Alzheimer's Disease. Neurobiol. Dis. 8, 890-899. doi: $10.1006 /$ nbdi.2001.0422
Reiman, E. M., Arboleda-Velasquez, J. F., Quiroz, Y. T., Huentelman, M. J., Beach, T. G., Caselli, R. J., et al. (2020). Exceptionally low likelihood of Alzheimer's dementia in APOE2 homozygotes from a 5,000-person neuropathological study. Nat. Commun. 11:667.

Ries, M., and Sastre, M. (2016). Mechanisms of A $\beta$ Clearance and Degradation by Glial Cells. Front. Aging Neurosci. 8:160.

Rudenko, G., Henry, L., Henderson, K., Ichtchenko, K., Brown, M. S., Goldstein, J. L., et al. (2002). Structure of the LDL receptor extracellular domain at endosomal pH. Science 298, 2353-2358. doi: 10.1126/science.1078124

Saeed, A. A., Genové, G., Li, T., Lütjohann, D., Olin, M., Mast, N., et al. (2014). Effects of a disrupted blood-brain barrier on cholesterol homeostasis in the brain. J. Biol. Chem. 289, 23712-23722. doi: 10.1074/jbc.m114.556159

Sakai, J., Nohturfft, A., Cheng, D., Ho, Y. K., Brown, M. S., and Goldstein, J. L. (1997). Identification of complexes between the COOH-terminal domains of sterol regulatory element-binding proteins (SREBPS) and SREBP cleavageactivating protein. J. Biol. Chem. 272, 20213-20221. doi: 10.1074/jbc.272.32. 20213

Sakai, J., Nohturfft, A., Goldstein, J. L., and Brown, M. S. (1998). Cleavage of sterol regulatory element-binding proteins (SREBPs) at site- 1 requires interaction with SREBP cleavage-activating protein. Evidence from in vivo competition studies. J. Biol. Chem. 273, 5785-5793. doi: 10.1074/jbc.273.10.5785

Saunders, A. M., Strittmatter, W. J., Schmechel, D., George-Hyslop, P. H., PericakVance, M. A., and Joo, S. H. (1993). Association of apolipoprotein E allele ?4 with late-onset familial and sporadic alzheimer's disease. Neurology 43, 1467-1472.

Scheltens, P., Blennow, K., Breteler, M. M. B., de Strooper, B., Frisoni, G. B., Salloway, S., et al. (2016). Alzheimer's disease [Internet]. Lancet 388, 505-517.

Schneider, A., Rajendran, L., Honsho, M., Gralle, M., Donnert, G., Wouters, F., et al. (2008). Flotillin-dependent clustering of the amyloid precursor protein regulates its endocytosis and amyloidogenic processing in neurons. J. Neurosci. 28, 2874-2882. doi: 10.1523/jneurosci.5345-07.2008

Schneider, A., Schulz-Schaeffer, W., Hartmann, T., Schulz, J. B., and Simons, M. (2006). Cholesterol depletion reduces aggregation of amyloid-beta peptide in hippocampal neurons. Neurobiol. Dis. 23, 573-577. doi: 10.1016/j.nbd.2006.04. 015

Schönknecht, P., Lütjohann, D., Pantel, J., Bardenheuer, H., Hartmann, T., von Bergmann, K., et al. (2002). Cerebrospinal fluid 24S-hydroxycholesterol is increased in patients with Alzheimer's disease compared to healthy controls. Neurosci. Lett. 324, 83-85. doi: 10.1016/s0304-3940(02)00164-7

Segarra, M., Aburto, M. R., and Acker-Palmer, A. (2021). Blood-Brain Barrier Dynamics to Maintain Brain Homeostasis. Trends Neurosci. 0:0.

Shi, Y., and Holtzman, D. M. (2018). Interplay between innate immunity and Alzheimer disease: APOE and TREM2 in the spotlight. Nat. Rev. Immunol. 18, 759-772. doi: 10.1038/s41577-018-0051-1

Shibuya, Y., Niu, Z., Bryleva, E. Y., Harris, B. T., Murphy, S. R., Kheirollah, A., et al. (2015). Acyl-coenzyme A: CHolesterol acyltransferase 1 blockage enhances autophagy in the neurons of triple transgenic Alzheimer's disease mouse and reduces human P301L-tau content at the presymptomatic stage. Neurobiol. Aging. 36, 2248-2259. doi: 10.1016/j.neurobiolaging.2015.04.002

Shimabukuro, M. K., Langhi, L. G. P., Cordeiro, I., Brito, J. M., Batista, C. M. D. C., Mattson, M. P., et al. (2016). Lipid-laden cells differentially distributed in the aging brain are functionally active and correspond to distinct phenotypes. Sci. Rep. 2016:6.

Simons, M., Keller, P., De Strooper, B., Beyreuther, K., Dotti, C. G., and Simons, K. (1998). Cholesterol depletion inhibits the generation of -amyloid in hippocampal neurons. Proc. Natl. Acad. Sci. 95, 6460-6464. doi: 10.1073/pnas. 95.11 .6460

Söderberg, M., Edlund, C., Kristensson, K., and Dallner, G. (1990). Lipid Compositions of Different Regions of the Human Brain During Aging. J. Neurochem. 54, 415-423. doi: 10.1111/j.1471-4159.1990.tb01889.x

Sodero, A. O. (2020). 24S-hydroxycholesterol: Cellular effects and variations in brain diseases [Internet]. J. Neurochem. 2020:24.

Solomon, A., Kivipelto, M., Wolozin, B., Zhou, J., and Whitmer, R. A. (2009). Midlife serum cholesterol and increased risk of Alzheimer's and vascular dementia three decades later. Dement Geriatr. Cogn. Disord. 28, 75-80. doi: $10.1159 / 000231980$

Song, Y., Hustedt, E. J., Brandon, S., and Sanders, C. R. (2013). Competition between homodimerization and cholesterol binding to the C99 domain of 
the amyloid precursor protein. Biochemistry 52, 5051-5064. doi: 10.1021/ bi400735x

Srinivasan, K., Friedman, B. A., Etxeberria, A., Huntley, M. A., van der Brug, M. P., Foreman, O., et al. (2020). Alzheimer's Patient Microglia Exhibit Enhanced Aging and Unique Transcriptional Activation. Cell Rep. 31, 107843. doi: 10. 1016/j.celrep.2020.107843

Stampfer, M. J. (2006). Cardiovascular disease and Alzheimer's disease: common links. J. Intern. Med. 260, 211-223. doi: 10.1111/j.1365-2796.2006.01687.x

Staurenghi, E., Cerrato, V., Gamba, P., Testa, G., Giannelli, S., Leoni, V., et al. (2021). Oxysterols present in Alzheimer's disease brain induce synaptotoxicity by activating astrocytes: A major role for lipocalin-2. Redox Biol. 1:39.

Strittmatter, W. J., Saunders, A. M., Schmechel, D., Pericak-Vance, M., Enghild, J., Salvesen, G. S., et al. (1993). Apolipoprotein E: High-avidity binding to $\beta$ amyloid and increased frequency of type 4 allele in late-onset familial Alzheimer disease. Proc. Natl. Acad. Sci. U S A. 90, 1977-1981. doi: 10.1073/pnas.90.5.1977

Tajima, Y., Ishikawa, M., Maekawa, K., Murayama, M., Senoo, Y., NishimakiMogami, T., et al. (2013). Lipidomic analysis of brain tissues and plasma in a mouse model expressing mutated human amyloid precursor protein/tau for Alzheimer's disease. Lipids Health Dis. 12:68. doi: 10.1186/1476-511x-12-68

Tarasoff-Conway, J. M., Carare, R. O., Osorio, R. S., Glodzik, L., Butler, T., Fieremans, E., et al. (2015). Clearance systems in the brain-implications for Alzheimer disease. Nat. Rev. Neurol. 11, 457-470.

Thambisetty, M., Simmons, A., Velayudhan, L., Hye, A., Campbell, J., Zhang, Y., et al. (2010). Association of plasma clusterin concentration with severity, pathology, and progression in Alzheimer disease. Arch. Gen. Psychiatry 67, 739-748.

Thelen, K. M., Falkai, P., Bayer, T. A., and Lütjohann, D. (2006). Cholesterol synthesis rate in human hippocampus declines with aging. Neurosci. Lett. 403, 15-19. doi: 10.1016/j.neulet.2006.04.034

Thrupp, N., Sala Frigerio, C., Wolfs, L., Skene, N. G., Fattorelli, N., Poovathingal, S., et al. (2020). Single-Nucleus RNA-Seq Is Not Suitable for Detection of Microglial Activation Genes in Humans. Cell Rep. 32:13.

Tini, G., Scagliola, R., Monacelli, F., La Malfa, G., Porto, I., Brunelli, C., et al. (2020). Alzheimer's Disease and Cardiovascular Disease: A Particular Association. Cardiol. Res. Pract. 2020:2617970.

Tomioka, M., Toda, Y., Mañucat, N. B., Akatsu, H., Fukumoto, M., Kono, N., et al. (2017). Lysophosphatidylcholine export by human ABCA7. Biochim. Biophys. Acta - Mol. Cell Biol. Lipids 1862, 658-665. doi: 10.1016/j.bbalip.2017.03.012

Ulrich, J. D., and Holtzman, D. M. (2016). TREM2 Function in Alzheimer's Disease and Neurodegeneration [Internet]. ACS Chem. Neurosci. 7, 420-427. doi: 10.1021/acschemneuro.5b00313

van der Kant, R., Langness, V. F., Herrera, C. M., Williams, D. A., Fong, L. K., Leestemaker, Y., et al. (2019). Cholesterol Metabolism Is a Druggable Axis that Independently Regulates Tau and Amyloid- $\beta$ in iPSC-Derived Alzheimer's Disease Neurons. Cell Stem Cell 24, 363.e-375.e.

Vance, J. E., and Hayashi, H. (2010). Formation and function of apolipoprotein E-containing lipoproteins in the nervous system. Biochim. Biophys. Acta-Mol. Cell Biol. Lipids 1801, 806-818. doi: 10.1016/j.bbalip.2010.02.007

Waelsch, H., Sperry, W. M., and Stoyanoff, V. A. (1940). Lipid Metabolism in Brain During Myelination. New York, NY: Columbia University.

Wahrle, S., Das, P., Nyborg, A. C., McLendon, C., Shoji, M., Kawarabayashi, T., et al. (2002). Cholesterol-dependent $\gamma$-secretase activity in buoyant cholesterolrich membrane microdomains. Neurobiol. Dis. 9, 11-23. doi: 10.1006/nbdi. 2001.0470

Wang, H., Kulas, J. A., Ferris, H. A., and Hansen, S. B. (2020). Regulation of beta-amyloid production in neurons by astrocyte-derived cholesterol. bioRxiv 2020:159632.

Whitmer, R. A., Gunderson, E. P., Barrett-Connor, E., Quesenberry, C. P., and Yaffe, K. (2005). Obesity in middle age and future risk of dementia: a 27 year longitudinal population based study. BMJ 330:1360. doi: 10.1136/bmj.38446. 466238.e0

Widenmaier, S. B., Snyder, N. A., Nguyen, T. B., Arduini, A., Lee, G. Y., Arruda, A. P., et al. (2017). NRF1 Is an ER Membrane Sensor that Is Central to Cholesterol Homeostasis. Cell 171, .e15-.e1109.

Wolozin, B., Kellman, W., Ruosseau, P., Celesia, G. G., and Siegel, G. (2000). Decreased prevalence of Alzheimer disease associated with 3-hydroxy-3methyglutaryl coenzyme A reductase inhibitors. Arch. Neurol. 57, 1439-1443. doi: 10.1001/archneur.57.10.1439
Wong, M. Y., Lewis, M., Doherty, J. J., Shi, Y., Cashikar, A. G., Amelianchik, A., et al. (2020). 25-Hydroxycholesterol amplifies microglial IL-1 $\beta$ production in an apoE isoform-dependent manner. J. Neuroinflam. 17:192.

Xie, C., Lund, E. G., Turley, S. D., Russell, D. W., and Dietschy, J. M. (2003). Quantitation of two pathways for cholesterol excretion from the brain in normal mice and mice with neurodegeneration. J. Lipid Res. 44:9.

Xiong, H., Callaghan, D., Jones, A., Walker, D. G., Lue, L. F., Beach, T. G., et al. (2008). Cholesterol retention in Alzheimer's brain is responsible for high $\beta$ and $\gamma$-secretase activities and A $\beta$ production. Neurobiol. Dis. 29, 422-437. doi: 10.1016/j.nbd.2007.10.005

Xu, Q., Bernardo, A., Walker, D., Kanegawa, T., Mahley, R. W., and Huang, Y. (2006). Profile and regulation of apolipoprotein E (ApoE) expression in the CNS in mice with targeting of green fluorescent protein gene to the ApoE locus. J. Neurosci. 26:19.

Yang, D.-S., Stavrides, P., Saito, M., Kumar, A., Rodriguez-Navarro, J. A., Pawlik, M., et al. (2014). Defective macroautophagic turnover of brain lipids in the TgCRND8 Alzheimer mouse model: prevention by correcting lysosomal proteolytic deficits. Brain 137, 3300-3318. doi: 10.1093/brain/awu278

Yao, Z., and Papadopoulos, V. (2002). Function of $\beta$-amyloid in cholesterol transport: a lead to neurotoxicity. FASEB J. 16, 1677-1679. doi: 10.1096/fj.020285fje

Yeh, F. L., Wang, Y., Tom, I., Gonzalez, L. C., and Sheng, M. (2016). TREM2 Binds to Apolipoproteins, Including APOE and CLU/APOJ, and Thereby Facilitates Uptake of Amyloid-Beta by Microglia. Neuron 2016:16.

Yuan, P., Condello, C., Keene, C. D., Wang, Y., Bird, T. D., Paul, S. M., et al. (2016). TREM2 Haplodeficiency in Mice and Humans Impairs the Microglia Barrier Function Leading to Decreased Amyloid Compaction and Severe Axonal Dystrophy. Neuron. 90, 724-739. doi: 10.1016/j.neuron.2016.05.003

Zhang, C., and Liu, P. (2017). The lipid droplet: A conserved cellular organelle [Internet]. Prot. Cell 8, 796-800. doi: 10.1007/s13238-017-0467-6

Zhang, J., and Liu, Q. (2015). Cholesterol metabolism and homeostasis in the brain. Protein Cell 6, 254-264. doi: 10.1007/s13238-014-0131-3

Zhang, X., Wen, J., and Zhang, Z. (2018). Statins use and risk of dementia: A dose-response meta analysis. Medicine 97:e11304. doi: 10.1097/md. 0000000000011304

Zhang, Y., Chen, K., Sloan, S. A., Bennett, M. L., Scholze, A. R., Phatnani, H. P., et al. (2014). Cellular/Molecular An RNA-Sequencing Transcriptome and Splicing Database of Glia, Neurons, and Vascular Cells of the Cerebral Cortex. J. Neurosci. 34, 11929-11947. doi: 10.1523/jneurosci.1860-14.2014

Zhao, J., Davis, M. D., Martens, Y. A., Shinohara, M., Graff-Radford, N. R., Younkin, S. G., et al. (2017). APOE $\varepsilon 4 / \varepsilon 4$ diminishes neurotrophic function of human iPSC-derived astrocytes. Hum. Mol. Genet. 26, 2690-2700. doi: 10.1093/hmg/ddx155

Zheng, H., and Koo, E. H. (2011). Biology and pathophysiology of the amyloid precursor protein. Mole. Neurodegener. 6:27. doi: 10.1186/1750-1326-6-27

Zhou, Y., Song, W. M., Andhey, P. S., Swain, A., Levy, T., Miller, K. R., et al. (2020). Human and mouse single-nucleus transcriptomics reveal TREM2-dependent and TREM2-independent cellular responses in Alzheimer's disease. Nat. Med. 26, 131-142. doi: 10.1038/s41591-019-0695-9

Zissimopoulos, J. M., Barthold, D., Brinton, R. D., and Joyce, G. (2017). Sex and Race Differences in the Association Between Statin Use and the Incidence of Alzheimer Disease. JAMA Neurol. 74, 225-232. doi: 10.1001/jamaneurol.2016. 3783

Zou, G., Su, J., Zhang, Y., Li, F., Li, C., and Meng, X. (2020). Effects of environmental enrichment on cognitive behavior and the expression of adenosine triphosphate binding cassette transporter A7 in hippocampus of adolescent mice with high fat diet. J. Cent. South Univ. 45, 892-900.

Conflict of Interest: The authors declare that the research was conducted in the absence of any commercial or financial relationships that could be construed as a potential conflict of interest.

Copyright $(2) 2021$ Feringa and van der Kant. This is an open-access article distributed under the terms of the Creative Commons Attribution License (CC BY). The use, distribution or reproduction in other forums is permitted, provided the original author(s) and the copyright owner(s) are credited and that the original publication in this journal is cited, in accordance with accepted academic practice. No use, distribution or reproduction is permitted which does not comply with these terms. 